BRX-TH 486

\title{
Partial Masslessness of Higher Spins in (A)dS
}

\author{
S. Deser and A. Waldron \\ Physics Department, Brandeis University, Waltham, MA 02454, USA \\ deser, wally@brandeis.edu
}

\begin{abstract}
Massive spin $s \geq 3 / 2$ fields can become partially massless in cosmological backgrounds. In the plane spanned by $m^{2}$ and $\Lambda$, there are lines where new gauge invariances permit intermediate sets of higher helicities, rather than the usual flat space extremes of all $2 s+1$ massive or just 2 massless helicities. These gauge lines divide the $\left(m^{2}, \Lambda\right)$ plane into unitarily allowed or forbidden intermediate regions where all $2 s+1$ massive helicities propagate but lower helicity states can have negative norms. We derive these consequences for $s=3 / 2,2$ by studying both their canonical (anti)commutators and the transmutation of massive constraints to partially massless Bianchi identities. For $s=2$, a Hamiltonian analysis exhibits the absence of zero helicity modes in the partially massless sector. For $s=5 / 2,3$ we derive Bianchi identities and their accompanying gauge invariances for the various partially massless theories with propagating helicities $( \pm 5 / 2, \pm 3 / 2)$ and $( \pm 3, \pm 2),( \pm 3, \pm 2, \pm 1)$, respectively. Of these, only the $s=3$ models are unitary. To these ends, we also provide the half integer generalization of the integer spin wave operators of Lichnerowicz. Partial masslessness applies to all higher spins in (A)dS as seen by their degree of freedom counts. Finally a derivation of massive $d=4$ constraints by dimensional reduction from their $d=5$ massless Bianchi identity ancestors is given.
\end{abstract}

PACS numbers: 03.65.Pm, 03.70.+k, 04.62.+v, 04.65.+e 


\section{Introduction}

The $\left(m^{2}, \Lambda\right)$ mass-cosmological constant plane for spin $s=2$ is unexpectedly rich [1, 2, 3]: For generic $\left(m^{2}, \Lambda\right)$, the theory describes 5 massive degrees of freedom. However, the field equations, having two open indices, are susceptible to both single and double divergence Bianchi identities each signaling the onset of gauge invariances. This is indeed realized when $m^{2}=0$ and $m^{2}=2 \Lambda / 3$, respectively. The former corresponds to linearized cosmological Einstein gravity and the latter to the partially massless de Sitter (dS) theory of [1]. A peculiarity of (Anti) de Sitter ((A)dS) spaces is that gauge invariance does not necessarily imply null propagation, which in fact occurs only for the $m^{2}=2 \Lambda / 3$ theory [1]. Furthermore, these two gauge invariant theories lie on the boundary of two physically distinct regions (see Figure 1). As demonstrated in [2], in the $\mathrm{dS}$ region with masses bounded by $0<m^{2}<2 \Lambda / 3$, the massive $s=2$ spectrum is not unitary. This is an example of a generic difficulty encountered by higher spin theories in backgrounds; it was first observed in a study of canonical anticommutators for charged massive $s=3 / 2$ fields in electromagnetic backgrounds [4] and later shown to imply acausal propagation [5].

The above $s=2$ behaviors are common spins $s \geq 3 / 2$ in (A)dS, as seen by studying possible Bianchi identities. The number of massive higher spin covariant field components exceeds the $2 s+1$ propagating degrees of freedom (PDoF) count. The correct count is imposed by constraints constructed from divergences of each open index of the field equations, $D^{\mu_{1}} \cdots D^{\mu_{n}} \mathcal{G}_{\mu_{1} \ldots \mu_{n} \mu_{n+1} \ldots}$ $+\cdots=0$. In flat space, all these constraints hold identically when the mass $m$ vanishes and are encapsulated by a single Bianchi identity $\partial^{\mu_{1}} \mathcal{G}_{\mu_{1} \mu_{2} \ldots}+\cdots=$ 0 (for $s>2$ the terms $+\cdots$ ensure (gamma-)tracelessness of the remaining open indices) reflecting the gauge invariance of the massless theory [6]. Once a cosmological constant is present, this degeneracy is broken and for special tunings of $m^{2}$ to $\Lambda$, intermediate situations are possible where some of the lower divergence constraints remain and all the higher ones are replaced by a single Bianchi identity [3]. This new identity entails a gauge invariance of the theory. As a consequence, higher spins in (A)dS spaces can be partially massless: not only are there strictly massive theories with all $2 s+1$ helicities and strictly massless ones with only helicities $\pm s$, but also intermediate theories of helicities $( \pm s, \pm(s-1), \ldots, \pm(s-t))(t<s)$ 円.

\footnotetext{
${ }^{1}$ An additional subtlety for $s \geq 5 / 2$ is that auxiliary fields are necessary (over and
} 

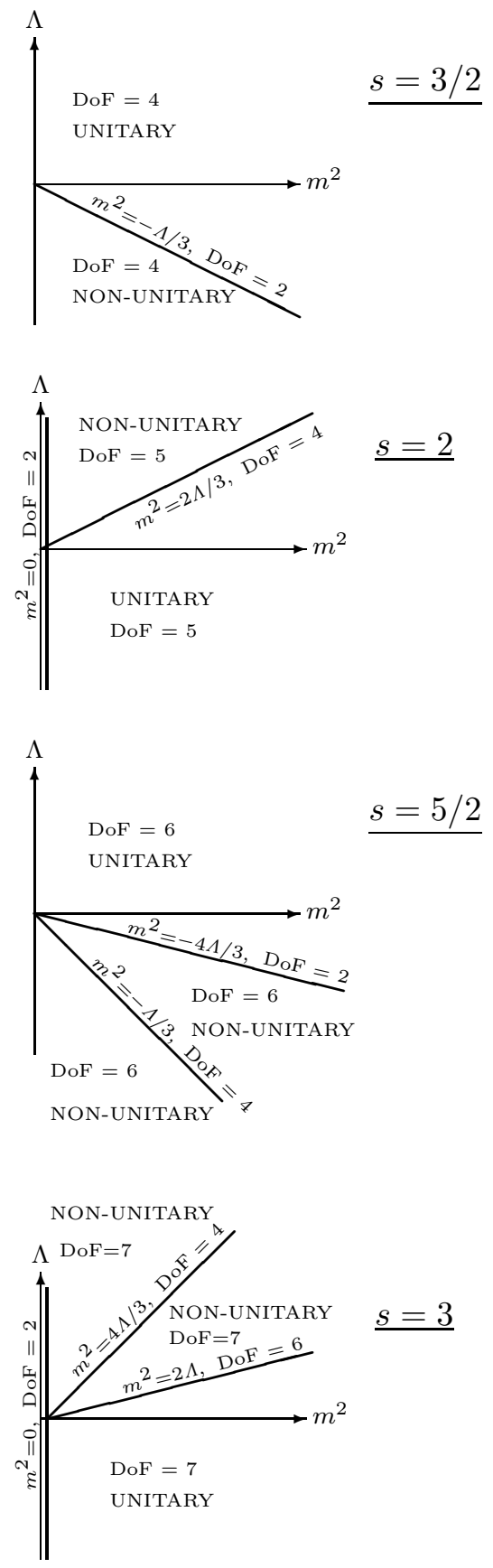

Figure 1: Respective phase diagrams for spins $s=3 / 2,2,5 / 2,3$ showing partially massless gauge lines and unitarily allowed and forbidden regions. 
The new Bianchi identities appear along lines in the $\left(m^{2}, \Lambda\right)$ plane and are also manifested in the canonical (anti)commutators of the fields. These follow directly from the covariant transverse-traceless decomposition of the on-shell fields [3]. The gauge lines in the $\left(m^{2}, \Lambda\right)$ plane correspond to poles in the lower helicity terms of (anti)commutators, the usual indication that a theory enjoys a gauge invariance at special values of parameters. Therefore, lower helicity (anti)commutators flip sign across these gauge lines. The intermediate regions correspond to (unitarily allowed or forbidden) massive theories; the one including the massive Minkowski theory is always unitary. The norms of those helicities that are removed by a gauge invariance flip sign across the corresponding line. This implies that the partially massless theories of the remaining helicities are unitary only when, starting from the unitary Minkowski region, the line in the $\left(m^{2}, \Lambda\right)$ half-plane removing the lowest helicity state(s) can be traversed first and subsequent lines are encountered in order, ending on the strictly massless helicity $\pm s$ gauge line. The latter is guaranteed to be unitary since it removes all dangerous lower helicities. We show explicitly that this condition is fulfilled for $s=2,3$ but not for the novel $s=5 / 2$ partially massless gauge line. We will also demonstrate, by a simple counting argument, that partial masslessness is enjoyed by all higher spins in $(\mathrm{A}) \mathrm{dS}$.

In Section 2 we introduce massive spins $s \leq 2$ and their gauge invariances. We also summarize the higher integer spin wave operators of Lichnerowicz [7] and provide their generalizations to half integer spins. In Section 3, we study canonical (anti)commutators for $s \leq 2$ and show how non-unitary regions arise. In Section \&, we examine the partially massless $m^{2}=2 \Lambda / 3, s=2$ theory, demonstrating by Hamiltonian analysis that it describes propagating helicities $( \pm 2, \pm 1)$ only. Section 5 deals with all spins: We first present a counting argument indicating that partial masslessness occurs for all higher spins in (A)dS. Then we display explicit new Bianchi identities and partially massless gauge invariances for $s=5 / 2,3$. The Appendix extends the dimensional reduction derivation of massive higher spin field equations in flat space from their massless dimension $d=5$ predecessors [8], to obtain massive constraints from the $d=5$ Bianchi identity for $s=5 / 2,3$ examples. Some

above the field content of the strictly massless models), but at least in the massless limit, these can be shown to decouple.

${ }^{2}$ Their restriction to equal time commutators agrees with the detailed $s=2$ Dirac analysis of 2 . 
future directions and open problems are discussed in the Conclusion. A brief version of some of our results was given in [3].

\section{Field Equations and Identities in Constant Curvature Spaces}

The Riemann tensor in constant curvature spaces is

$$
R_{\mu \nu \rho \sigma}=-\frac{2 \Lambda}{3} g_{\mu[\rho} g_{\sigma] \nu}
$$

the cosmological constant $\Lambda$ is positive in $\mathrm{dS}$ and negative in AdS. The actions of commutators of covariant derivatives are summarized by the vector-spinor examplef

$$
\left[D_{\mu}, D_{\nu}\right] \psi_{\rho}=\frac{2 \Lambda}{3} g_{\rho[\mu} \psi_{\nu]}+\frac{\Lambda}{6} \gamma_{\mu \nu} \psi_{\rho}
$$

The actions and field equations for massive spins $0 \leq s \leq 2$ in constant curvature backgroundsf are

$$
\begin{aligned}
& \mathcal{L}^{(0)}=\frac{1}{2} \sqrt{-g} \phi \mathcal{G}, \\
& \mathcal{L}^{(1)}=\frac{1}{2} \sqrt{-g} \phi^{\mu} \mathcal{G}_{\mu}, \\
& \mathcal{L}^{(2)}=\frac{1}{2} \sqrt{-g} \phi^{\mu \nu} \mathcal{G}_{\mu \nu}, \\
& \mathcal{G}=\left(\Delta^{(0)}-m^{2}\right) \phi, \\
& \mathcal{R}=\left(\mathcal{D}^{(1 / 2)}+m\right) \psi, \\
& \mathcal{G}_{\mu}=\left(\Delta^{(1)}-m^{2}\right) \phi_{\mu}-D_{\mu} D \cdot \phi, \\
& \mathcal{R}_{\mu} \equiv \gamma_{\mu \nu \rho} \mathcal{D}^{\nu} \psi^{\rho}=\left(\mathcal{D}^{(3 / 2)}-\not D\right) \psi_{\mu}-m \gamma_{\mu \nu} \psi^{\nu} \text {, } \\
& \mathcal{G}_{\mu \nu} \equiv\left(\Delta^{(2)}-m^{2}+\Lambda\right)\left(\phi_{\mu \nu}-g_{\mu \nu} \phi_{\rho}{ }^{\rho}\right)+\Lambda \phi_{\mu \nu} \\
& +D_{(\mu} D_{\nu)} \phi_{\rho}^{\rho}-2 D_{(\mu} D . \phi_{\nu)}+g_{\mu \nu} \text { D.D. } \phi \text {. }
\end{aligned}
$$

\footnotetext{
${ }^{3}$ Our metric is "mostly plus", Dirac matrices are "mostly hermitean" and the Dirac conjugate is $\bar{\psi} \equiv \psi^{\dagger} i \gamma^{0}$. We denote (anti)symmetrization with unit weight by round (resp. square) brackets. Antisymmetrized products of Dirac matrices are given by $\gamma^{\mu_{1} \ldots \mu_{n}} \equiv$ $\gamma^{\left[\mu_{1}\right.} \cdots \gamma^{\left.\mu_{n}\right]}$.

${ }^{4}$ For $s=2$ in generic gravitational backgrounds, the minimally coupled Pauli-Fierz action does not yield field equations with the correct $2 s+1=5$ PDoF [9]. Recently [10], $s=2$ actions have been constructed with the correct PDoF count in background Einstein spaces $\left(R_{\mu \nu}=\Lambda g_{\mu \nu}\right)$.
} 
The operators $\Delta^{(n)}$ are the wave operators of [7]

$$
\begin{aligned}
\Delta^{(3)} \phi_{\mu \nu \rho} & \equiv D^{2} \phi_{\mu \nu \rho}-5 \Lambda \phi_{\mu \nu \rho}+2 \Lambda g_{(\mu \nu} \phi_{\rho) \sigma}{ }^{\sigma}, \\
\Delta^{(2)} \phi_{\mu \nu} & \equiv D^{2} \phi_{\mu \nu}-\frac{8 \Lambda}{3}\left(\phi_{\mu \nu}-\frac{1}{4} g_{\mu \nu} \phi_{\rho}{ }^{\rho}\right) \\
\Delta^{(1)} \phi_{\mu} & \equiv\left(D^{2}-\Lambda\right) \phi_{\mu} \\
\Delta^{(0)} \phi & \equiv D^{2} \phi
\end{aligned}
$$

whose introduction is justified by the following identities,

$$
\begin{aligned}
\Delta^{(3)} D_{(\mu} \phi_{\nu \rho)} & =D_{(\mu} \Delta^{(2)} \phi_{\nu \rho)}, & D^{\mu} \Delta^{(3)} \phi_{\mu \nu \rho} & =\Delta^{(2)} D . \phi_{\nu \rho}, \\
\Delta^{(3)} g_{(\mu \nu} \phi_{\rho)} & =g_{(\mu \nu} \Delta^{(1)} \phi_{\rho)}, & g^{\mu \nu} \Delta^{(3)} \phi_{\mu \nu \rho} & =\Delta^{(1)} \phi_{\sigma \rho}^{\sigma}, \\
\Delta^{(2)} D_{(\mu} \phi_{\nu)} & =D_{(\mu} \Delta^{(1)} \phi_{\nu)}, & D^{\mu} \Delta^{(2)} \phi_{\mu \nu} & =\Delta^{(1)} D . \phi_{\nu}, \\
\Delta^{(2)} g_{\mu \nu} \phi & =g_{\mu \nu} \Delta^{(0)} \phi, & & g^{\mu \nu} \Delta^{(2)} \phi_{\mu \nu}=\Delta^{(0)} \phi_{\rho}^{\rho}, \\
\Delta^{(1)} D_{\mu} \phi & =D_{\mu} \Delta^{(0)} \phi, & D^{\mu} \Delta^{(1)} \phi_{\mu} & =\Delta^{(0)} D . \phi .
\end{aligned}
$$

Here $\phi_{\mu \nu \ldots}$ is a symmetric tensor. The half integer spin generalizations $\mathcal{D}^{(n / 2)}$ of these operators are given by [11]

$$
\begin{aligned}
\mathcal{D}^{(5 / 2)} \psi_{\mu \nu} & \equiv 3 \not D \psi_{\mu \nu}-2 D_{(\mu} \gamma \cdot \psi_{\nu)}+2 \gamma_{(\mu}\left(\not D \gamma \cdot \psi_{\nu)}-D \cdot \psi_{\nu)}\right) \\
\mathcal{D}^{(3 / 2)} \psi_{\mu} & \equiv 2 \not D \psi_{\mu}-D_{\mu} \gamma \cdot \psi+\gamma_{\mu}(\not D \gamma . \psi-D . \psi)=\gamma_{\mu \nu \rho} D^{\nu} \psi^{\rho}+\not D \psi_{\mu} \\
\mathcal{D}^{(1 / 2)} \psi & \equiv \not D \psi
\end{aligned}
$$

with $\psi_{\mu \nu}$ a symmetric spinor-tensor, and satisfy analogous identities

$$
\begin{aligned}
\mathcal{D}^{(5 / 2)} D_{(\mu} \psi_{\nu)} & =D_{(\mu} \mathcal{D}^{(3 / 2)} \psi_{\nu)}, & D^{\mu} \mathcal{D}^{(5 / 2)} \psi_{\mu \nu} & =\mathcal{D}^{(3 / 2)} D \cdot \psi_{\nu}, \\
\mathcal{D}^{(5 / 2)} \gamma_{(\mu} \psi_{\nu)} & =\gamma_{(\mu} \mathcal{D}^{(3 / 2)} \psi_{\nu)}, & \gamma^{\mu} \mathcal{D}^{(5 / 2)} \psi_{\mu \nu} & =\mathcal{D}^{(3 / 2)} \gamma \cdot \psi_{\nu}, \\
\mathcal{D}^{(3 / 2)} D_{\mu} \psi & =D_{\mu} \mathcal{D}^{(1 / 2)} \psi, & D^{\mu} \mathcal{D}^{(3 / 2)} \psi_{\mu} & =\mathcal{D}^{(1 / 2)} D . \psi, \\
\mathcal{D}^{(3 / 2)} \gamma_{\mu} \psi & =\gamma_{\mu} \mathcal{D}^{(1 / 2)} \psi, & \gamma^{\mu} \mathcal{D}^{(3 / 2)} \psi_{\mu} & =\mathcal{D}^{(1 / 2)} \gamma \cdot \psi .
\end{aligned}
$$

For completeness we include the familiar Weitzenbock identity,

$$
\left(\mathcal{D}^{(1 / 2)}\right)^{2} \psi=\left(D^{2}-\Lambda\right) \psi .
$$


Notice that we have written the massive Rarita-Schwinger field equation (7) in terms of the $s=3 / 2$ operator $\mathcal{D}^{(3 / 2)}$ with explicit mass term as well as in a more compact form involving the operator

$$
\mathcal{D}_{\mu} \equiv D_{\mu}+\frac{m}{2} \gamma_{\mu}, \quad\left[\mathcal{D}_{\mu}, \mathcal{D}_{\nu}\right]=\left[D_{\mu}, D_{\nu}\right]+\left(m^{2} / 2\right) \gamma_{\mu \nu},
$$

encountered in cosmological supergravity [12].

For $s \geq 1$ there are more relativistic field components than PDoF. As usual, the correct PDoF count is obtained by studying the constraints implied by divergences and (gamma-)traces of the field equations. Less usual, for special lines in the $\left(\mathrm{m}^{2}, \Lambda\right)$ plane, these constraints are satisfied identically and become Bianchi identities associated with gauge invariances. Explicitly, the divergence of the field equations (6)-(8) read

$$
\begin{gathered}
D . \mathcal{G}=-m^{2} D . \phi, \\
D . \mathcal{G}_{\nu}=-m^{2}\left(D . \phi_{\nu}-D_{\nu} \phi\right)
\end{gathered}
$$

and are constraints for generic values of the parameters $m^{2}$ and $\Lambda$. For $s=1,2$, the value $m^{2}=0$ yields Bianchi identities and their associated ("electromagnetic" and "general coordinate") gauge invariances

$$
\delta \phi_{\mu}=D_{\mu} \xi \quad \text { and } \quad \delta \phi_{\mu \nu}=D_{(\mu} \xi_{\nu)}
$$

respectively. Both $m^{2}=0$ theories are strictly massless: they propagate with two physical helicity states. For $s=3 / 2$, the sole gauge invariance

$$
\delta \psi_{\mu}=\mathcal{D}_{\mu} \varepsilon=D_{\mu} \varepsilon+\frac{1}{2} \sqrt{-\Lambda / 3} \gamma_{\mu} \varepsilon
$$

is inherited from cosmological supergravity and occurs for $m^{2}=-\Lambda / 3$ in AdS. This (rather than the $m=0$ model) is the strictly massless helicity $\pm 3 / 2$ theory.

For $s=2$ there is a new effect: the field equation has two open indices and thereby also admits a double divergence Bianchi identity. The double divergence constraint

$$
D . D . \mathcal{G}+\frac{1}{2} m^{2} \mathcal{G}_{\rho}{ }^{\rho}=\frac{1}{2} m^{2}\left(3 m^{2}-2 \Lambda\right) \phi_{\rho}{ }^{\rho},
$$


becomes a Bianchi identity not only along the strictly massless line $m^{2}=0$, but also along the dS gauge line $m^{2}=2 \Lambda / 3$ corresponding to the Weyl-like theory of [1]. The relevant gauge invariance

$$
\delta \phi_{\mu \nu}=\left(D_{(\mu} D_{\nu)}+\frac{\Lambda}{3} g_{\mu \nu}\right) \xi
$$

may be employed to show that this partially massless model propagates with helicities $( \pm 2, \pm 1)$ on the null cone in $\mathrm{dS}$. We discuss this theory further in Section 1 .

To summarize, the $\left(m^{2}, \Lambda\right)$ half-plane offers no surprises for spins $s \leq 1$, the usual null propagating massless theories inhabit the line $m^{2}=0$ and all other points $m^{2} \geq 0$ describe $2 s+1$ massive DoF. For $s=3 / 2$, the massless line is $m^{2}=-\Lambda / 3$ and bisects the $\left(m^{2}, \Lambda\right)$ half-plane as depicted in Figure 1 . For $s=2$, the massless theory again lies on the axis $m^{2}=0$ and a new gauge invariance emerges at $m^{2}=2 \Lambda / 3$ which also divides the half-plane into two distinct physical regions.

\section{3 (Anti)commutators and Nonunitary Regions}

Our analysis is rather simple and harks back to the original inconsistency of the local field theory of charged spin 3/2 particles [4. Generically, given (anti)commutation relations $\left\{\psi, \psi^{\dagger}\right\}=\epsilon=\left[a, a^{\dagger}\right]=-i[x, \dot{x}]$ (with $a=$ $(x+i \dot{x}) / \sqrt{ } 2)$ and a vacuum $\psi|0\rangle=0=a|0\rangle$, positivity of norms requires $\epsilon>0$. For quantum field theories, exactly the same criterion can be applied, but now in a distributional sense.

The local canonical (anti)commutators for quantum fields with $s \leq 2$ in cosmological spaces were presented long ago [7]. Let us summarize those results: For $s=0,1 / 2$ one has

$$
\begin{aligned}
{\left[\phi(x), \phi\left(x^{\prime}\right)\right] } & =i D^{(0)}\left(x, x^{\prime} ; m^{2}\right), \\
\left\{\psi(x), \bar{\psi}\left(x^{\prime}\right)\right\} & =i S^{(1 / 2)}\left(x, x^{\prime} ; m\right) .
\end{aligned}
$$

The distributions on the right hand sides of (25) and (26) are general coordinate/local Lorentz biscalars and bivectors [14], respectively, and share the

\footnotetext{
${ }^{5}$ It has recently been suggested that the non-perturbative definition of quantum gravity in dS be reexamined [13]; in particular the definition of the vacuum requires careful consideration. Our local quantum field theoretic computation ignores such subtleties.
} 
symmetry properties of the (anti)commutators on the left hand sides

$$
D^{(0)}\left(x, x^{\prime}\right)=-D^{(0)}\left(x, x^{\prime}\right), \quad S^{(1 / 2)}\left(x, x^{\prime}\right) \gamma^{0}=-\gamma^{0}\left[S^{(1 / 2)}\left(x^{\prime}, x\right)\right]^{\dagger} .
$$

We often drop the the labels " $m$ " and " $m$ " used to indicate that these distributions satisfy the field equations of the onshell quantum fields $\phi(x)$ and $\psi(x)$

$$
\begin{gathered}
\left(\Delta_{x}^{(0)}-m^{2}\right) D^{(0)}\left(x, x^{\prime} ; m^{2}\right)=0=\left(\Delta_{x^{\prime}}^{(0)}-m^{2}\right) D^{(0)}\left(x, x^{\prime} ; m^{2}\right), \\
\left(\mathcal{D}^{(1 / 2)}+m\right) S^{(1 / 2)}\left(x, x^{\prime} ; m\right)=0=S^{(1 / 2)}\left(x, x^{\prime} ; m\right)\left(-\overleftarrow{\mathcal{D}}_{x^{\prime}}^{(1 / 2)}+m\right)
\end{gathered}
$$

Here the backwards arrow over an operator $\mathcal{O}$ is defined by

$$
\bar{\psi} \overleftarrow{\mathcal{O}} \equiv-\overline{\mathcal{O} \psi}
$$

The boundary conditions for these distributions are

$$
\begin{gathered}
\left.\frac{d}{d x^{\prime 0}} D^{(0)}\left(x, x^{\prime}\right)\right|_{x^{0}=x^{\prime 0}}=\frac{1}{\sqrt{-g}} \delta^{3}\left(\vec{x}-\vec{x}^{\prime}\right),\left.\quad D^{(0)}\left(x, x^{\prime}\right)\right|_{x^{0}=x^{\prime 0}}=0 \\
\left.S^{(1 / 2)}\left(x, x^{\prime}\right)\right|_{x^{0}=x^{\prime 0}}=\frac{\gamma^{0}}{\sqrt{-g}} \delta^{3}\left(\vec{x}-\vec{x}^{\prime}\right)
\end{gathered}
$$

for any choice of timelike coordinate $x^{0}$.

Note that in general, the (anti)commutator distributions can be written as the difference of advanced and retarded propagators [7]. In the Minkowski limit, the scalar distribution $D^{(0)}\left(x, x^{\prime} ; m^{2}\right)$ is the usual Pauli-Jordan commutator function.

For $s \geq 1$, away from the gauge invariant boundaries, the constraints (20) and (23) imply the (gamma-)traceless-transverse conditions

$$
\begin{gathered}
D . \phi=0, \\
D . \phi_{\nu}=0=\phi_{\rho}{ }^{\rho},
\end{gathered}
$$

which allow the field equations (6)-(8) to be rewritten as

$$
\begin{gathered}
\left(\Delta^{(1)}-m^{2}\right) \phi_{\mu}=0, \\
\left(\Delta^{(2)}-m^{2}+2 \Lambda\right) \phi_{\mu \nu}=0 .
\end{gathered}
$$


We must now write (anti)commutators for fields satisfying both (32) and (33). The latter requirement is just the analog of the $s=0,1 / 2$ solutions given above. The former is easily imposed using the (gamma-)traceless-transverse decompositions

$$
\begin{gathered}
\phi_{\mu}^{\mathrm{T}}=\phi_{\mu}-D_{\mu} \frac{1}{D^{2}} D \cdot \phi \\
D \cdot \phi^{\mathrm{T}}=0 ; \\
\psi_{\mu}^{\mathrm{TT}}=\psi_{\mu}-\frac{1}{4} \gamma_{\mu} \gamma \cdot \psi+D_{\widetilde{\mu}} \frac{1}{3 D^{2}+\Lambda}(\not D \gamma \cdot \psi-4 D \cdot \psi), \\
D \cdot \psi^{\mathrm{TT}}=0=\gamma \cdot \psi^{\mathrm{TT}} ; \\
\phi_{\mu \nu}^{\mathrm{TT}}=\phi_{\mu \nu}-D_{(\mu} \frac{2}{D^{2}+\Lambda}\left(D \cdot \phi_{\nu)}\right)^{\mathrm{T}}-\frac{1}{4} g_{\mu \nu} \phi_{\rho}{ }^{\rho} \\
-D_{\{\mu} D_{\nu\}} \frac{4}{D^{2}\left(3 D^{2}+4 \Lambda\right)}\left[D \cdot D \cdot \phi-\frac{1}{4} D^{2} \phi_{\rho}{ }^{\rho}\right], \\
D \cdot \phi_{\mu}^{\mathrm{TT}}=0=\phi_{\rho}^{\mathrm{TT} \rho} ;
\end{gathered}
$$

where a tilde over an index denotes its gamma-traceless part, i.e. $X_{\widetilde{\mu}} \equiv X_{\mu}-$ $\frac{1}{4} \gamma_{\mu} \gamma . X$. and $\{. \cdot\}$ denotes the symmetric-traceless part of any symmetric tensor, i.e. $X_{\{\mu \nu\}} \equiv X_{(\mu \nu)}-\frac{1}{4} g_{\mu \nu} X_{\rho}{ }^{\rho}$.

Therefore the (anti)commutators for spins $1 \leq s \leq 2$ are given by

$$
\begin{aligned}
{\left[\phi_{\mu}(x), \phi_{\nu}\left(x^{\prime}\right)\right]=} & i D_{\mu \nu}^{(1)}\left(x, x^{\prime} ; m^{2}\right)+\frac{i}{m^{2}} D_{\mu}^{x} D_{\nu}^{x^{\prime}} D^{(0)}\left(x, x^{\prime} ; m^{2}\right) \\
\left\{\psi_{\mu}(x), \bar{\psi}_{\nu}\left(x^{\prime}\right)\right\}= & i S_{\mu \nu}^{(3 / 2)}\left(x, x^{\prime} ; 2 m\right)-\frac{i}{4} \gamma_{\mu}^{x} S^{(1 / 2)}\left(x, x^{\prime} ; 2 m\right) \gamma_{\nu}^{x^{\prime}} \\
& +\frac{i}{3 m^{2}+\Lambda} \mathcal{D}_{\mu}^{x} S^{(1 / 2)}\left(x, x^{\prime} ; 2 m\right) \overleftarrow{\mathcal{D}}_{\nu}^{x^{\prime}} \\
{\left[\phi_{\mu \nu}(x), \phi_{\rho \sigma}\left(x^{\prime}\right)\right]=} & i D_{\mu \nu, \rho \sigma}^{(2)}\left(x, x^{\prime} ; m^{2}-2 \Lambda\right)+\frac{2 i}{m^{2}} D_{\mu}^{x} D_{\rho}^{x^{\prime}} D_{\nu \sigma}^{(1)}\left(x, x^{\prime} ; m^{2}-2 \Lambda\right) \\
+ & \frac{i}{m^{2}\left(3 m^{2}-2 \Lambda\right)}\left[2 D_{\mu}^{x} D_{\nu}^{x} D_{\rho}^{x^{\prime}} D_{\sigma}^{x^{\prime}}+m^{2}\left(\Lambda-m^{2}\right) g_{\mu \nu}^{x} g_{\rho \sigma}^{x^{\prime}}\right. \\
& \left.+m^{2} D_{\mu}^{x} D_{\nu}^{x} g_{\rho \sigma}^{x^{\prime}}+m^{2} g_{\mu \nu}^{x} D_{\rho}^{x^{\prime}} D_{\sigma}^{x^{\prime}}\right] D^{(0)}\left(x, x^{\prime} ; m^{2}-2 \Lambda\right)
\end{aligned}
$$


For brevity, we have suppressed obvious symmetrizations $(\mu \nu)$ and $(\rho \sigma)$ on the right hand side of (39). The field equations (33) have been used throughout to eliminate any factors $D^{2}$ appearing in the (gamma-)tracelesstransverse decompositions (34)-(36), since the higher distributions are also onshell

$$
\begin{aligned}
\left(\Delta^{(n)}+m^{2}\right) D_{\mu_{1} \ldots \mu_{n}, \nu_{1} \ldots \nu_{n}}^{(n)}\left(x, x^{\prime} ; m^{2}\right) & \equiv 0, \\
\left(\mathcal{D}^{(n / 2)}+m\right) S_{\mu_{1} \ldots \mu_{n}, \nu_{1} \ldots \nu_{n}}^{(n / 2)}\left(x, x^{\prime} ; m\right) & \equiv 0 .
\end{aligned}
$$

The identity $D_{\widetilde{\mu}}^{x} S^{(1 / 2)}\left(x, x^{\prime} ; 2 m\right)=\mathcal{D}_{\mu}^{x} S^{(1 / 2)}\left(x, x^{\prime} ; 2 m\right)$ is also useful. The (anti)commutators (37)-(39) are the difference between advanced and retarded propagators $\left[7\right.$. The higher distributions $D^{(1)}, D^{(2)}$ and $S^{(3 / 2)}$ above satisfy

$$
\begin{aligned}
& D_{x^{\prime}}^{\nu} D_{\mu \nu}^{(1)}\left(x, x^{\prime}, m^{2}\right)=-D_{\mu}^{x} D^{(0)}\left(x, x^{\prime} ; m^{2}\right), \\
& S_{\mu \nu}^{(3 / 2)}\left(x, x^{\prime} ; m\right) \overleftarrow{\mathcal{D}}_{x^{\prime}}^{\nu}=-\mathcal{D}_{\mu}^{x} S^{(1 / 2)}\left(x, x^{\prime} ; m\right), \\
& S_{\mu \nu}^{(3 / 2)}\left(x, x^{\prime} ; m\right) \gamma_{x^{\prime}}^{\nu}=\gamma_{\mu}^{x} S^{(1 / 2)}\left(x, x^{\prime} ; m\right), \\
& D_{x^{\prime}}^{\rho} D_{\mu \nu, \rho \sigma}^{(2)}\left(x, x^{\prime}, m^{2}\right)=-D_{(\mu}^{x} D_{\nu \sigma)}^{(1)}\left(x, x^{\prime} ; m^{2}\right), \\
& g_{x^{\prime}}^{\rho \sigma} D_{\mu \nu, \rho \sigma}^{(2)}\left(x, x^{\prime}, m^{2}\right)=g_{\mu \nu}^{x} D^{(0)}\left(x, x^{\prime}, m^{2}\right),
\end{aligned}
$$

with boundary conditions

$$
\begin{gathered}
\left.\frac{d}{d x^{\prime 0}} D_{\mu \nu}^{(1)}\left(x, x^{\prime}\right)\right|_{x^{0}=x^{\prime 0}}=\frac{g_{\mu \nu}}{\sqrt{-g}} \delta^{3}\left(\vec{x}-\vec{x}^{\prime}\right),\left.\quad D_{\mu \nu}^{(1)}\left(x, x^{\prime}\right)\right|_{x^{0}=x^{\prime 0}}=0, \\
\left.S_{\mu \nu}^{(3 / 2)}\left(x, x^{\prime}\right)\right|_{x^{0}=x^{\prime 0}}=\frac{g_{\mu \nu} \gamma^{0}}{\sqrt{-g}} \delta^{3}\left(\vec{x}-\vec{x}^{\prime}\right) . \\
\left.\frac{d}{d x^{\prime 0}} D_{\mu \nu, \rho \sigma}^{(2)}\left(x, x^{\prime}\right)\right|_{x^{0}=x^{\prime 0}}=\frac{\left.g_{(\mu(\rho)} g_{\sigma) \nu}\right)}{\sqrt{-g}} \delta^{3}\left(\vec{x}-\vec{x}^{\prime}\right),\left.\quad D_{\mu \nu, \rho \sigma}^{(2)}\left(x, x^{\prime}\right)\right|_{x^{0}=x^{\prime 0}}=0 .
\end{gathered}
$$

Equipped with the above tools, one can easily uncover the nonunitary regions. Starting with the (anti)commutators (37)-(39), the aim is to determine whether the distributions on their right hand sides have definite sign in the dangerous lower helicity sectors.

For concreteness we work in the simple synchronous dS metric

$$
d s^{2}=-d t^{2}+e^{2 M t} d \vec{x}^{2}, \quad M \equiv \sqrt{\Lambda / 3},
$$


and concentrate on the equal time (anti)commutators of the time components of the fields (and their time derivatives). While the metric (44) does not cover the entire $\mathrm{dS}$ space, nor is it real when continued to negative AdS values of $\Lambda$, these disadvantages are outweighed by its simplicity (we will consider more general coordinate systems later). Selecting the lowest helicity components by looking at time components of fields, a simple computation reveals that

$$
\begin{aligned}
{\left[\phi_{0}(t, \vec{x}), \dot{\phi}_{0}\left(t, \vec{x}^{\prime}\right)\right] } & =-\frac{\nabla^{2}}{m^{2}} \frac{i}{\sqrt{-g}} \delta^{3}\left(\vec{x}-\vec{x}^{\prime}\right), \\
\left\{\psi_{0}(t, \vec{x}), \psi_{0}^{\dagger}\left(t, \vec{x}^{\prime}\right\}\right. & =-\frac{\nabla^{2}}{3 m^{2}+\Lambda} \frac{1}{\sqrt{-g}} \delta^{3}\left(\vec{x}-\vec{x}^{\prime}\right), \\
{\left[\phi_{00}(t, \vec{x}), \dot{\phi}_{00}\left(t, \vec{x}^{\prime}\right)\right] } & =\frac{2 \nabla^{4}}{m^{2}\left(3 m^{2}-2 \Lambda\right)} \frac{i}{\sqrt{-g}} \delta^{3}\left(\vec{x}-\vec{x}^{\prime}\right),
\end{aligned}
$$

where $\nabla^{2} \equiv g^{i j} \partial_{i} \partial_{j}=e^{-2 M t} \vec{\partial}^{2}$ is a negative operator. The final equation (47) agrees with the detailed massive $s=2$ Dirac analysis presented in [2]. Our derivation only requires writing out the Laplace and Dirac operators explicitly in the metric (44) and using the field equations (33) to maximally eliminate time derivatives, after which the boundary conditions (31) and (43) may be applied.

The interpretation of equations (45)-(47) is as follows: Positivity of the distributions on the right hand sides is completely determined by the respective denominators $m^{2}, 3 m^{2}+\Lambda$ and $3 m^{2}-2 \Lambda$, precisely the factors appearing in the Bianchi identities of the previous Section. For the various spins, we learn:

- Spin 1: The model is unitary in the entire $\left(m^{2}, \Lambda\right)$ half-plane. A line of gauge invariant models emerges at $m^{2}=0$ (the same value as in flat space).

- Spin 3/2: The model is unitary in the region $m^{2}>-\Lambda / 3$ which includes the Minkowski background. Strictly massless, gauge invariant unitary models are found along the AdS line $m^{2}=-\Lambda / 3$. The region $m^{2}<-\Lambda / 3$ is non-unitary. In contrast to flat space, the $m^{2}=0$ theories are massive when $\Lambda \neq 0$ and even non-unitary for negative (AdS) values of $\Lambda$.

- Spin 2: Models with $m^{2}>2 \Lambda / 3$ are unitary. There are now two lines of gauge invariant theories; the usual linearized cosmological Einstein 
theory at $m^{2}=0$ and a partially massless theory [1] at $m^{2}=2 \Lambda / 3$. Both are unitary but the region $m^{2}<2 \Lambda / 3$ is not.

These conclusions are depicted in Figure 11.

Finally, as promised, we address the concern that, strictly, the metric (44) applies only to $\mathrm{dS}$. On the one hand, given that (i) the final results are a function of the real variable $\Lambda$ only and (ii) the picture presented here is backed up by the emergence of Bianchi identities, there can be no doubt of its correctness. However, for complete certainty, we repeat, as an example, the $s=3 / 2$ computation in the metric $(M \equiv \sqrt{\Lambda / 3})$

$$
d s^{2}=-d t^{2}+\cosh ^{2}(M t)\left\{d r^{2}+\frac{1}{M^{2}} \sin ^{2}(M r)\left(d \theta^{2}+\sin ^{2} \theta d \phi^{2}\right)\right\} .
$$

Upon rescaling $r \rightarrow \rho / M$, the three-metric $d \Omega^{2}=d \rho^{2}+\sin ^{2} \rho\left(d \theta^{2}+\sin ^{2} \theta d \phi^{2}\right)$ is seen to describe a unit three-sphere. We prefer the initial parametrization, however, since for pure imaginary values of $M$, the cosmological constant $\Lambda$ is negative and the metric continues to AdS. Performing a calculation analogous to the one above we find

$$
\begin{aligned}
& \left\{\psi_{0}(t, r, \theta, \phi), \psi_{0}^{\dagger}\left(t, r^{\prime}, \theta^{\prime}, \phi^{\prime}\right)\right\}= \\
& \quad \frac{\cosh ^{-2}(M t)\left(-{ }^{(3)} D^{2}-\Lambda / 4\right)}{3 m^{2}+\Lambda} \frac{1}{\sqrt{-g}} \delta\left(r-r^{\prime}\right) \delta\left(\theta-\theta^{\prime}\right) \delta\left(\phi-\phi^{\prime}\right) .
\end{aligned}
$$

The operator ${ }^{(3)} D^{2}$ is the square of the intrinsic 3-dimensional covariant derivative (Laplace-Beltrami operator) acting on a spinor. In dS the operator $-{ }^{(3)} D^{2}-\Lambda / 4$ is not manifestly positive. However (in our parametrization) the eigenvalues of ${ }^{(3)} D^{2}$ acting on spinors are $(\Lambda / 3)(-l(l+2)+1 / 2)$ with $l \geq 1 / 2$ (see, e.g. [2]), and the highest eigenvalue is precisely $-\Lambda / 4$. Hence the operator $-{ }^{(3)} D^{2}-\Lambda / 4$ is indeed positive and in $\mathrm{dS}$ we may draw precisely the conclusions given above. Now, continuing the metric (48) to AdS space the same result holds for the local anticommutator except the 3-space is a hyperboloid. Nonetheless, (assuming we can neglect spatial boundary terms), both $-{ }^{(3)} D^{2}$ and $-\Lambda / 4$ are now separately positive, and unitarity is determined by the sign of the denominator $3 \Lambda+m^{2}$. This concludes our derivation of the unitarily forbidden regions for spins $s \leq 2$.

We emphasize that once one knows the gauge lines and their corresponding Bianchi identities, our unitarity results in fact follow by inspection: 
Whenever a coefficient in a massive constraint vanishes and then becomes negative, all the corresponding lower helicity modes are first excised by the accompanying gauge invariance and thereafter reemerge with opposite norms. Therefore, starting from the unitary Minkowski region, it is easy to map out the unitarily allowed and forbidden regions, as shown in Figure 11. Furthermore, for higher spin partially massless theories to be unitary, the ordering criterion for the gauge lines, discussed in the introduction, must hold. A simple example is provided by the $s=2$ strictly massless $\left(m^{2}=0\right.$, linearized graviton) theory for $\Lambda>0$ : To reach it starting from the unitary Minkowski region, one must pass through the unitarily forbidden region $0<m^{2}<2 \Lambda / 3$. Nonetheless, the theory is unitary, since the highest helicities \pm 2 are left untouched by the unitarity flip of the helicity 0 mode across the $m^{2}=2 \Lambda / 3$ gauge line.

\section{Partially Massless Spin 2: Canonical Analysis}

At the partially massless dS boundary, $m^{2}=2 \Lambda / 3=2 M^{2}$, we showed that the scalar constraint (23) is a Bianchi identity; as such it removes 2 DoF, leaving 4 physical DoF corresponding to helicities $\pm 2, \pm 1$. In this Section we prove this claim via an explicit canonical analysiso? Our method is similar to that originally used to prove the stability of massless cosmological gravity [17].

A possible starting point is the second order massive $s=2$ action in equation (5). Equivalently (and much simpler) one can begin with the first order ADM form of cosmological Einstein gravity,

$$
\begin{array}{r}
S_{\Lambda+E}=\int d^{4} x\left[\pi^{i j} \dot{g}_{i j}+N \sqrt{g}\left({ }^{(3)} R-2 \Lambda\right)+2 N_{i} D_{j} \pi^{i j}\right. \\
\left.+\frac{N}{\sqrt{g}} \pi^{i j} \pi^{l m}\left(\frac{1}{2} g_{i j} g_{l m}-g_{i l} g_{j m}\right)\right],
\end{array}
$$

then linearize around a dS background and add by hand an explicit mass term. Here $g$ is the determinant of the 3-metric $g_{i j}$ and $N \equiv\left(-g^{00}\right)^{-1 / 2}$, $N_{0 i} \equiv g_{0 i}$. We take the synchronous dS metric (44), denoted $d s^{2}=-d t^{2}+$ $\bar{g}_{i j} d x^{i} d x^{j}$ in this Section, reserving $g_{i j}$ for the dynamical 3-metric which we

\footnotetext{
${ }^{6}$ A detailed canonical analysis of massive $s=2$ for general $m^{2}$ is given in 15]; an early attempt can be found in 16 .
} 
linearize as

$$
g_{i j} \equiv \bar{g}_{i j}+\phi_{i j}, \quad \bar{g}_{i j} \equiv f^{2}(t) \delta_{i j}, \quad f(t) \equiv e^{M t}
$$

The remaining fields are linearized as

$$
\pi^{i j} \equiv \bar{\pi}^{i j}+P^{i j}, \quad \bar{\pi}^{i j} \equiv-2 M f \delta^{i j}, \quad N \equiv 1+\widetilde{n} .
$$

(The background metric is block diagonal so no expansion is needed for $N_{i}$.) In terms of these deviations, the mass term is

$$
S_{m}=-\frac{m^{2}}{4} \int d^{4} x \sqrt{\bar{g}}\left(\phi_{\mu \nu} \phi_{\rho \sigma} \bar{g}^{\mu \rho} \bar{g}^{\nu \sigma}-\left(\phi_{\mu \nu} \bar{g}^{\mu \nu}\right)^{2}\right),
$$

here $\phi_{0 i} \equiv N_{i}, \bar{g} \equiv \operatorname{det} \bar{g}_{i j}=-\operatorname{det} \bar{g}_{\mu \nu}$ and $\phi_{00} \equiv g_{00}+\bar{g}_{00}=-\left(1+N^{2}\right)$. The final action is the sum $S=S_{\Lambda+E}+S_{m}$, discarding any terms of higher than quadratic order in (dynamical) fields.

Notice that the only explicit time dependence of the integrand of (53) is through $f^{-1}$. Indeed it proves useful to make the field redefinition

$$
\begin{aligned}
\phi_{i j} & \equiv f^{1 / 2} h_{i j}, \quad P^{i j} \equiv f^{-1 / 2} p^{i j} . \\
N_{i} \equiv f^{1 / 2} n_{i}, & \widetilde{n}=f^{-3 / 2} n .
\end{aligned}
$$

The cost is an extra contribution generated by the symplectic term of (50), $P^{i j} \dot{\phi}_{i j} \rightarrow p^{i j} \dot{h}_{i j}+(M / 2) p^{i j} h_{i j}$. A dividend is that the only explicit time dependence in what follows will be through $\nabla^{2} \equiv \bar{g}^{i j} \partial_{i} \partial_{j} \equiv f^{-2} \nabla_{0}^{2}$. Index contractions are just with $\delta_{i j}$, all quantities are now in $(3+1)$ form.

Next examine the mass term

$$
S_{m}=\int d^{4} x\left[-\frac{m^{2}}{4}\left(h_{i j}^{2}-h_{i i}^{2}\right)+\frac{m^{2}}{2} N_{i}^{2}+m^{2} n h_{i i}\right] .
$$

Were it not for the term proportional to $N_{i}^{2}$, the field $N_{i}$ would be a Lagrange multiplier for 3 constraints (as is the case for the $m=0$ strictly massless theory). Instead, when $m \neq 0$ we must integrate out $N_{i}$ via its algebraic equation of motion. The field $n$, however, only appears linearly and remains a Lagrange multiplier for the constraint

$$
\left[\sqrt{g}\left({ }^{(3)} R-2 \Lambda\right)+m^{2} h_{i i}+\frac{1}{\sqrt{g}} \pi^{i j} \pi^{l m}\left(\frac{1}{2} g_{i j} g_{l m}-g_{i l} g_{j m}\right)\right]_{\text {linearized }}=0 .
$$


For generic values of $\left(m^{2}, \Lambda\right)$, this constraint eliminates one degree of freedom from the 6 pairs $\left(p^{i j}, h_{i j}\right)$ leaving 5 physical helicities $( \pm 2, \pm 1,0)$. Our aim now is to show that a (single) further constraint emerges at the gauge invariant value $m^{2}=2 M^{2}$.

We decompose the fields $h_{i j}$ and $p_{i j}$ according to their helicity and drop (for now) the helicity \pm 2 traceless-transverse $\left(h_{i j}^{t t}, p_{i j}^{t t}\right)$ and helicity \pm 1 transverse $\left(h_{i}^{t}, p_{i}^{t}\right)$ modes since they manifestly decouple from the 2 remaining helicity 0 modes (to the quadratic order used here). The latter are defined by the projection

$$
h_{i j}=\frac{1}{2}\left(\delta_{i j}-\frac{\partial_{i} \partial_{j}}{\nabla_{0}^{2}}\right) h_{T}+\frac{\partial_{i} \partial_{j}}{\nabla_{0}^{2}} h_{L},
$$

and similarly for $p_{i j}$. (Note that under the integral $\int A_{i j} B_{i j}=\frac{1}{2} \int A_{T} B_{T}+$ $\left.\int A_{L} B_{L}.\right)$ In terms of these variables the linearized constraint is

$$
\mathcal{C} \equiv \nabla^{2} h_{T}+2 M\left(p_{T}+p_{L}\right)-\left(m^{2}-2 M^{2}\right)\left(h_{T}+h_{L}\right)=0 .
$$

Note that the leading term comes from the linearized 3-dimensional curvature scalar and that both the cosmological $-2 \Lambda \sqrt{g}$ and momentum squared terms in (56) contribute to the final term in (58), which vanishes on the critical gauge line $m^{2}=2 M^{2}$. Henceforth we concentrate on the critical case and eliminate $m^{2}$ via this relation.

Next we write out the quadratic action $S=S_{E+\Lambda}+S_{m}$ remembering the constraint (58) which we solve as

$$
p_{T}=-p_{L}-\frac{\nabla^{2}}{2 M} h_{T} .
$$

Observe that the symplectic terms become

$$
p_{i j} \dot{h}_{i j}=\frac{1}{2} p_{T} \dot{h}_{T}+p_{L} \dot{h}_{L}=p_{L} \dot{q}-\frac{1}{4} h_{T} \nabla^{2} h_{T}, \quad q \equiv h_{L}-\frac{1}{2} h_{T} .
$$

(suppressing integrations throughout). Upon eliminating $h_{L}=q+h_{T} / 2$ in favor of $q$ and $h_{T}$, the action depends only on the 3 variables $\left(p_{L}, q, h_{T}\right)$ and its most general form is

$$
S\left(p_{L}, q, h_{T}\right)-p_{L} \dot{q}=\frac{1}{2} A h_{T}^{2}+B h_{T}+C,
$$

where $A$ is constant, $B$ linear and $C$ quadratic in $\left(p_{L}, q\right)$. If $A=0$, we have an additional constraint $B\left(p_{L}, q\right)=0$ and no zero helicity PDoF remain, 
whereas for non-zero $A, h_{L}$ can be removed via its algebraic field equation leaving behind one zero helicity DoF. In fact, $A$ does vanish on the critical line $m^{2}=2 \Lambda / 3$ so this model describes helicities $( \pm 2, \pm 1)$ only. Indeed, a lengthy calculation yields

$$
S\left(p_{L}, q, h_{T}\right)-p_{L} \dot{q}=\frac{1}{2}\left(\frac{p_{L}}{M}-q\right) \nabla^{2} h_{T}+\left(\frac{p_{L}}{M}-q\right)\left[\left(\frac{\nabla^{2}}{m^{2}}-\frac{3}{2}\right) p_{L}-\left(\nabla^{2}-m^{2}\right) q\right] \text {. }
$$

As claimed, $A=0$ and even the zero helicity Hamiltonian vanishes once the Lagrange multiplier $h_{T}$ is integrated out.

Let us now examine the remaining helicities $( \pm 2, \pm 1)$. A series of canonical transformations yields a simple action

$$
S_{( \pm 2, \pm 1)}=\sum_{\varepsilon=( \pm 2, \pm 1)}\left\{p_{\varepsilon} \dot{q}_{\varepsilon}-\frac{1}{2}\left[p_{\varepsilon}^{2}+q_{\varepsilon}\left(-\nabla^{2}-\frac{M^{2}}{4}\right) q_{\varepsilon}\right]\right\} .
$$

Notice again, all time dependence is through $\nabla^{2}$ in the Hamiltonian. The field equations are

$$
p_{\varepsilon}=\dot{q}_{\varepsilon}, \quad\left(-\frac{d^{2}}{d t^{2}}+\nabla^{2}-\frac{M^{2}}{4}\right) q_{\varepsilon}=0 .
$$

The covariant field equation (33) evaluated at $m^{2}=2 M^{2}$ is $\left(D^{2}-4 M^{2}\right) \phi_{\mu \nu}=$ 0 . Consider, for example, helicities \pm 2 , for which $\partial^{i} \phi_{i j}=0=\phi_{i}{ }^{i}$. In this frame the transverse-traceless part of the covariant field equation reads

$$
\left(-\frac{d^{2}}{d t^{2}}+M \frac{d}{d t}+\nabla^{2}\right) \phi_{i j}=0 .
$$

The action (63) was obtained by the same rescaling as in (54), namely, $\phi_{i j}=$ $f^{1 / 2} q_{i j}$. The factor $f^{1 / 2}$ is precisely the integrating factor which removes the single time derivative from equation (65) at the cost of a term $-M^{2} / 4$, i.e. equations (65) and (64) are identical (helicities \pm 1 agree via a similar calculation).

Stability of the partially massless theory requires that it possess a conserved, positive, energy function. The latter can be obtained by an argument similar to that given in [17] for the strictly massless $s=2$ theory: The Hamiltonian in (63) is not conserved because of the explicit time dependence of $\nabla^{2}$. However, inside the intrinsic dS horizon at $\left(f M x^{i}\right)^{2}=1$, the background metric (44) possesses a timelike Killing vector

$$
\xi^{\mu}=\left(-1, M x^{i}\right) \Longrightarrow \xi^{2}=-1+\left(f M x^{i}\right)^{2} .
$$


Therefore, the energy associated with time evolution in this Killing direction

$$
E=T_{\mu}^{0} \xi^{\mu}=H-M x^{i}\left[p_{\varepsilon} \partial_{i} q_{\varepsilon}-\frac{1}{2} \partial_{i}\left(p_{\varepsilon} q_{\varepsilon}\right)\right]
$$

satisfies $\dot{E}=0$ ( $H$ is the Hamiltonian in (63) and we have suppressed the sum over helicities $\varepsilon$ ). Furthermore, writing out $E$ explicitly and relabeling the variable $p_{\varepsilon} \rightarrow p_{\varepsilon}+(3 M / 2) q_{\varepsilon}$ gives

$$
E=\frac{1}{2}\left(\widehat{x}^{i} p_{\varepsilon}\right)^{2}+\frac{1}{2}\left(f^{-1} \partial_{i} q_{\varepsilon}\right)^{2}-f M|x|\left(\widehat{x}^{i} p_{\varepsilon}\right)\left(f^{-1} \partial_{i} q_{\varepsilon}\right)+\frac{1}{2}\left(2 M^{2}\right) q_{\varepsilon}^{2},
$$

with $x^{i} \equiv|x| \widehat{x}^{i}$. The last (mass) term is manifestly positive and the first three terms are positive by the triangle equality whenever

$$
f M|x|<1,
$$

that is, inside the physically accessible region.

A final interesting feature of the partially massless $s=2$ theory is null propagation. The dS metric is conformally flat and it can be shown that the $m^{2}=2 \Lambda / 3$ theory propagates on its null cone [1]. An interesting open question is whether any of the $s>2$ partially massless theories which we discuss next, share this behavior.

\section{$5 \quad$ Higher Spins}

Having seen that the $s=2$ field equation $\mathcal{G}_{\mu \nu}$ yields both single divergence, $D . \mathcal{G}_{\nu}$, and double divergence, D.D.G. Bianchi identities we are led to inquire whether even higher divergence Bianchi identities occur for $s>2$. The answer is yes. This implies that, in addition to the usual massive and strictly massless possibilities, a spin $s$ field in (A)dS can be partially massless with propagating helicities $( \pm s, \pm(s-1), \ldots, \pm(s-t))(t<s)$. We first demonstrate this claim by simple counting arguments and then write explicit gauge invariances and Bianchi identities for $s=5 / 2,3$ examples.

\subsection{Higher Spin Bosons}

Define the number of components $(\underline{s})$ of a symmetric $s$-index tensor $\phi_{\mu_{1} \ldots \mu_{s}}$

$$
(\underline{s}) \equiv\left\{\begin{array}{cl}
\frac{(s+1)(s+2)(s+3)}{3 !} & s>0 \\
0 & \text { otherwise. }
\end{array}\right.
$$


Then a traceless symmetric tensor has $(\underline{s})^{\mathrm{T}}$ components

$$
(\underline{s})^{\mathrm{T}}=(\underline{s})-(\underline{s-2})\left(=(s+1)^{2}, s \geq 0\right),
$$

while a doubly traceless $\left(0=\phi_{\rho}^{\rho}{ }^{\sigma} \sigma \mu \ldots\right)$ one has

$$
(\underline{s})^{\mathrm{TT}}=(\underline{s})-(\underline{s-4})\left(=2\left(s^{2}+1\right), s \geq 1\right) \text {. }
$$

\section{Strictly Massless Bosons}

First consider massless bosons. The field content is a doubly traceless $s$ index symmetric tensor enjoying gauge invariances with an $s-1$ index traceless gauge parameter:

\begin{tabular}{|r|c|}
\hline Fields & $(\underline{s})^{\mathrm{TT}}$ \\
\hline \hline - Gauge & 2. $(\underline{\underline{s-1}})^{\mathrm{T}}$ \\
\hline
\end{tabular}

Since $2(\underline{s-1})^{\mathrm{T}}$ DoF can be gauged away, the PDoF for $s \geq 1$ are $(\underline{s})^{\mathrm{TT}}-$ $2(\underline{s-1})^{\mathrm{T}}=2$, corresponding to helicities $\pm s$.

\section{Massive Bosons}

For massive $s>2$ theories, the massless field content must be augmented by a set of traceless symmetric auxiliary fields, $\left(\chi, \ldots, \chi_{\mu_{1} \ldots \mu_{s-3}}\right)$. Each divergence of the $s$-index symmetric field equations is a constraint whenever the remaining open indices are traceless:

\begin{tabular}{|c|c|}
\hline $\begin{array}{c}\text { Fields } \\
+ \text { Auxiliaries }\end{array}$ & $(\underline{s})^{\mathrm{TT}}$ \\
$(\underline{0})^{\mathrm{T}}+\cdots+(\underline{s-3})^{\mathrm{T}}$ \\
\hline \hline - Constraints & $(\underline{0})^{\mathrm{T}}+\cdots+(\underline{\underline{s-3}})^{\mathrm{T}}+(\underline{\underline{s-2}})^{\mathrm{T}}+(\underline{\underline{s-1}})^{\mathrm{T}}$ \\
\hline
\end{tabular}

and the PDoF are $(\underline{s})^{\mathrm{TT}}-(\underline{s-2})^{\mathrm{T}}-(\underline{s-1})^{\mathrm{T}}=2 s+1$, the sum of all helicities.

\section{Partially Massless Bosons}

For partially massless higher spin theories the field content is the same as the massive case but new Bianchi identities appear. There are as many of these as possible divergences of the $s$-index symmetric field equations. On a gauge line where a constraint with $t$ divergences becomes a Bianchi identity we have: 


\begin{tabular}{|l|c|}
\hline $\begin{array}{l}\text { Fields } \\
+ \text { Auxiliaries }\end{array}$ & $(\underline{s})^{\mathrm{TT}}$ \\
\hline $\begin{array}{l}\text { - Constraints } \\
- \text { Gauge }\end{array}$ & $2 \cdot(\underline{s-t})^{\mathrm{T}}$ \\
\hline
\end{tabular}

The $t$-divergence Bianchi identity replaces the set of constraints with $t, t+$ $1, \ldots, s$ divergences and the corresponding gauge invariance removes $2 .(\underline{s-t})^{\mathrm{T}}$ DoF. Therefore we find $2 t \mathrm{PDoF}$ along with a set $\left((\underline{0})^{\mathrm{T}}+\cdots+(\underline{s-2-t})^{\mathrm{T}}\right)$ of leftover auxiliary fields. When the new Bianchi identity is the scalar one with the maximal number of divergences $t=s$, there are $2 s$ PDoF and only the massive helicity 0 mode is removed. For the vector Bianchi with $t=s-1$ there are $2 s-2$ PDoF because helicities $(0, \pm 1)$ are excised. For even lower values of $t$, the sum over leftover auxiliaries $(\underline{0})^{\mathrm{T}}+\cdots+(\underline{s-2-t})^{\mathrm{T}}$ is nonempty: In the strictly massless case $t=1$, there $2 \mathrm{PDoF}$ (helicities $\pm s$ ) and all the auxiliaries $(\underline{0})^{\mathrm{T}}+\cdots+(\underline{s-3})^{\mathrm{T}}$ remain. However, at least in this case, it is obvious that they decouple. Less obvious is whether they decouple for the partially massless $s \geq 4$ theories. If not, we must then ask how many PDoF they represent and whether they are unitary excitations, questions clearly inaccessible via the simple counting arguments presented here.

\subsection{Higher Spin Fermions}

For brevity define $\sigma \equiv s-1 / 2$. The (real) components of a $\sigma$-index, symmetric, gamma-traceless (Majorana) field are denoted

$$
\text { 4. }(\underline{\sigma})^{\gamma}=4[(\underline{\sigma})-(\underline{\sigma-1})](=2(\sigma+1)(\sigma+2), \sigma \geq 0) \text {, }
$$

and a traceless gamma-tracelessness one $\left(\gamma \cdot \psi^{\rho}{ }_{\rho \mu_{2} \ldots \mu_{\sigma}}=0\right)$ has

$$
\text { 4. }(\underline{\sigma})^{\gamma \mathrm{T}}=4\left[(\underline{\sigma})^{\gamma \mathrm{T}}-(\underline{\sigma-3})^{\gamma \mathrm{T}}\right] \quad\left(=2\left(3 s^{2}+3 s+2\right), \sigma \geq 0\right)
$$

components.

\section{Strictly Massless Fermions}

For massless half integer $s=\sigma+1 / 2$ the field content is a traceless gammatraceless, symmetric $\sigma$-index spinor. The correct number of PDoF is ensured by gauge invariances with a gamma-traceless, symmetric $(\sigma-1)$-index spinor parameter: 


\begin{tabular}{|rl|c|}
\hline $1 / 2$ & Fields & $4 .(\underline{\sigma})^{\gamma \mathrm{T}}$ \\
\hline$-1 / 2$ & Gauge & $4 .(\underline{\underline{\sigma-1}})^{\gamma}$ \\
\hline \hline- & Gauge & $4 .(\underline{\sigma-1})^{\gamma}$ \\
\hline
\end{tabular}

To explain: we begin with $4 .(\underline{\sigma})^{\gamma \mathrm{T}}$ fields, of which gauge invariance removes 4. $(\underline{\sigma-1})^{\gamma}$ components. However, before excising further DoF via residual invariances we must impose the projector field equations (recall that the Dirac equation divides the DoF of a spinor by 2). Thereafter residual gauge invariances remove 4. $(\underline{\sigma-1})^{\gamma}$ further DoF. Hence the PDoF count is $2\left[(\underline{\sigma})^{\gamma \mathrm{T}}-3(\underline{\sigma-1})^{\gamma}\right]=2$, corresponding to helicities $\pm s$.

\section{Massive Fermions}

In addition to the same fields as for massless fermions, there are traceless auxiliary spinor fields $\left(\chi, \ldots, \chi_{\mu_{1} \ldots \mu_{\sigma-2}}\right)$ when $s \geq 3 / 2$. All possible divergences on $\sigma$ vector indices of the field equations yield constraints when the leftover indices are gamma-traceless:

\begin{tabular}{|cl|c|}
\hline $1 / 2$ & Fields & $4 .(\underline{\sigma})^{\gamma \mathrm{T}}$ \\
\hline $1 / 2$ & Auxiliaries & $4 .(\underline{0})^{\mathrm{T}}+\cdots+4 .(\underline{\sigma-2})^{\mathrm{T}}$ \\
\hline \hline- & Constraints & $4 .(\underline{0})^{\gamma+\cdots+4 .(\underline{\sigma-1})^{\gamma}}$ \\
\hline
\end{tabular}

Adding these up yields $2 \sigma+2=2 s+1 \mathrm{PDoF}$ and all helicities $( \pm s, \ldots, \pm 1 / 2)$ propagate.

\section{Partially Massless Fermions}

The field content is identical to massive case but now suppose that $t$ divergences of the field equations yield a Bianchi identity. We have:

\begin{tabular}{|rl|c|}
\hline $1 / 2$ & Fields & $4 .(\underline{\sigma})^{\gamma \mathrm{T}}$ \\
\hline $1 / 2$ & Auxiliaries & $4 .(\underline{0})^{\mathrm{T}}+\cdots+4 .(\underline{\sigma-2})^{\mathrm{T}}$ \\
$-1 / 2$ & Gauge & $4 .(\underline{\sigma-t})^{\gamma}$ \\
\hline \hline- & Constraints & $4 \cdot(\underline{\underline{\sigma-t+1}})^{\gamma}+\cdots+4 .(\underline{\sigma-1})^{\gamma}$ \\
\hline- & Gauge & $4 .(\underline{\sigma-t})^{\gamma}$ \\
\hline
\end{tabular}

The sum is $2 t+2\left[(\underline{0})^{\mathrm{T}}+\cdots+(\underline{\sigma-t-1})^{\mathrm{T}}\right]$. Again we find $2 t$ propagating helicity states $( \pm s, \ldots, \pm(s-t))$ along with leftover auxiliaries which decouple on the strictly massless $(t=1)$ gauge line. (Just as in the bosonic case, for 
partially massless spins $s \geq 7 / 2$, little is known about the role of these auxiliaries).

We next present the explicit $s=5 / 2,3$ examples. For $s=5 / 2$ we will exhibit one new gauge line resulting from the fermionic generalization of the $s=2$ double divergence Bianchi identity (23). The resulting helicity $( \pm 5 / 2, \pm 3 / 2)$ partially massless theory lives in an AdS region where helicities $\pm 3 / 2$ have negative norms and therefore fails to be unitary. In contrast, for $s=3$ we find both double and triple divergence Bianchi identities corresponding to unitary, partially massless, $\mathrm{dS}$ theories of helicities $( \pm 3, \pm 2)$ and $( \pm 3, \pm 2, \pm 1)$.

\subsection{Spin $5 / 2$}

The $s=5 / 2$ spinorial field equation has two open indices, so as for $s=2$, there are two possible Bianchi identities; they appear along the AdS gauge lines $m^{2}=-4 \Lambda / 3$ and $m^{2}=-\Lambda / 3$ (see Figure 1). The former is the strictly massless theory with helicities $\pm 5 / 2$ whereas the novel gauge invariance of the latter removes only the lowest $\pm 1 / 2$ leaving helicities $( \pm 5 / 2, \pm 3 / 2)$. Since the massless gauge lines all lie in AdS (just as for their $s=3 / 2$ counterpart), the $\left(m^{2}, \Lambda\right)$ half-plane is divided into 3 regions. Only the $m^{2}>-4 \Lambda / 3$ one including Minkowski space, is unitary.

The $s=5 / 2$ action and field equations are

$$
\begin{gathered}
\mathcal{L}=-\sqrt{-g} \bar{\psi}^{\mu \nu} \mathcal{R}_{\mu \nu}-\sqrt{-g} \bar{\chi} \mathcal{R}_{5}, \\
\mathcal{R}_{\mu \nu}=\left(\mathcal{D}^{(5 / 2)}-2 \not D\right) \psi_{\mu \nu}+g_{\mu \nu} \gamma \cdot D \cdot \psi+\left(D_{(\mu} \gamma_{\nu)}-\frac{1}{2} g_{\mu \nu} \not D\right) \psi_{\rho}{ }^{\rho} \\
+m\left(\psi_{\mu \nu}-2 \gamma_{(\mu} \gamma \cdot \psi_{\nu)}-\frac{1}{2} g_{\mu \nu} \psi_{\rho}{ }^{\rho}\right)-\frac{5}{12} \mu g_{\mu \nu} \chi, \\
\mathcal{R}_{5}=-\alpha(\not D-3 m) \chi-\frac{5}{12} \mu \psi_{\rho}{ }^{\rho} .
\end{gathered}
$$

They can be derived by minimally coupling the flat space equations of Appendix A.1 to the cosmological background. The former were obtained by KK descent from their massless counterparts in $d=5$. Minimal coupling alone does not provide equations of motion describing the $6=2 s+1$ massive PDoF: An additional non-minimal coupling contained by the term $\mu \bar{\chi} \psi_{\rho}{ }^{\rho}$ is necessary. In fact, to achieve a proper set of constraints requires fixing the 
auxiliary coupling to

$$
\mu^{2}=\frac{12 \alpha}{5}\left(m^{2}+4 \Lambda / 3\right)
$$

Here we encounter a new subtlety. Implicitly we have so far assumed that physical models live in the half plane $m^{2}>0$, since for fermions negative $m^{2}$ implies a non-hermitean mass term, and for bosons, one that is unbounded below. While there are regions with both $m^{2}<0$ and the correct sign for anticommmutators, the dynamics is non-unitary there. Therefore we continue to require $m^{2}>0$ and examine the relation (79). Since hermiticity of the action (75) demands $\mu^{2}>0$, we find two regions: (i) $m^{2}>-4 \Lambda / 3>0$, (ii) $0<m^{2}<-4 \Lambda / 3$. Up until now, $\alpha$ was a free parameter which we could set to \pm 1 . In the region (i), $m^{2}+4 \Lambda / 3>0$ so we must take $\alpha=+1$. In region (ii), hermiticity of the action can be maintained at the cost of changing the sign of $\alpha$ to $\alpha=-1$ (the actions are then different in each region). In either case, we will find that region (ii) is unitarily forbidden.

Before continuing, it is interesting to compare these difficulties to $s=3 / 2$ and the problem of constructing $\mathrm{dS}$ supergravities [18]. As we have shown, $s=3 / 2$ is unitary for $m^{2} \geq-\Lambda / 3 \geq 0$ and the boundary is the strictly massless AdS theory corresponding to cosmological supergravity. As one follows the massive theory into $\mathrm{dS}$, the canonical anticommutators all have the correct sign for unitary representations. In fact, keeping $\Lambda>0$, there is no obstruction at the level of anticommutators to continuing to $m^{2}<0$ until the branch of the line $m^{2}=-\Lambda / 3$ with $m^{2}<0<\Lambda$ is reached. The theory there is formally supersymmetric (i.e. strictly massless) but the action is no longer hermitean, which is a example of the general statement that $\mathrm{dS}$ supergravities do not exist [18]. One might speculate that this clash is generic to higher spin fermions.

Returning to the $s=5 / 2$ field equations, for generic $\left(m^{2}, \Lambda\right)$ the constraints

$$
\begin{aligned}
\mathcal{C}_{\widetilde{\nu}} & \equiv D \cdot \mathcal{R}_{\widetilde{\nu}}+\frac{1}{4} m \gamma \cdot \mathcal{R}_{\widetilde{\nu}} \\
& =-\frac{5}{4}\left(m^{2}+4 \Lambda / 3\right) \gamma \cdot \psi_{\widetilde{\nu}}-\frac{5}{12} \mu D_{\widetilde{\nu}} \chi \\
\mathcal{C} & \equiv D \cdot \mathcal{C}+\frac{5}{16}\left(m^{2}+4 \Lambda / 3\right) \mathcal{R}_{\rho}{ }^{\rho}-\frac{5}{16} \alpha \mu(\not D+3 m) \mathcal{R}_{5} \\
& =-\frac{10}{3} \mu\left(m^{2}+\Lambda / 3\right) \chi
\end{aligned}
$$

ensure that the model describes $6=2 s+1$ massive PDoF. Along the AdS 
lines

$$
m^{2}=-4 \Lambda / 3 \quad m^{2}=-\Lambda / 3
$$

the constraints (81) and (83) transmute to Bianchi identities with respective (distinct) gauge invariances

$$
\begin{array}{rlrl}
\delta \psi_{\mu \nu}=D_{(\mu} \varepsilon_{\widetilde{\nu})}+\frac{1}{2} \sqrt{\frac{-\Lambda}{3}} \gamma_{(\mu} \varepsilon_{\widetilde{\nu})}, & & \delta \chi=0 \\
\delta \psi_{\mu \nu}=D_{(\mu} D_{\widetilde{\nu})} \varepsilon+\frac{5 \Lambda}{16} g_{\mu \nu} \varepsilon, & \delta \chi=-\frac{1}{8 \alpha} \sqrt{15 \alpha \Lambda}(\not D+\sqrt{-3 \Lambda}) \varepsilon .
\end{array}
$$

The vector-spinor Bianchi identity (80) at $m^{2}=-4 \Lambda / 3$ implies strict masslessness (propagating helicities $\pm 5 / 2$ ) since its invariance removes helicities $( \pm 3 / 2, \pm 1 / 2)$. Notice also that $\mu=0$ on the strictly massless line so, as claimed above, the spinor auxiliary $\chi$ decouples there. The novel spinor Bianchi identity (83) at $m^{2}=-\Lambda / 3$ and invariance (86) removes helicities $\pm 1 / 2$ leaving a partially massless theory of helicities $( \pm 5 / 2, \pm 3 / 2)$.

Once again, the coefficients $\left(m^{2}+4 \Lambda / 3\right)$ and $\left(m^{2}+\Lambda / 3\right)$ appearing in the constraints (80) and (83) control the positivity of equal time anticommutators. Therefore, since the gauge lines all lie in AdS, the $\left(m^{2}, \Lambda\right)$-plane is divided into 3 regions; only the one including Minkowski space $m^{2}>-4 \Lambda / 3$ is unitary. Although the strictly massless, $\mathrm{AdS}, \mathrm{m}^{2}=-4 \Lambda / 3$ theory is unitary, the partially massless one is not, as it fails the line ordering requirement: Starting from the unitary Minkowski region where all norms are positive, one would like first to traverse the line $m^{2}=-\Lambda / 3$, but that is only possible in dS with negative $m^{2}$ (imaginary values of $m$ violate hermiticity of the action and unitary evolution). Crossing the AdS strictly massless line $m^{2}=-4 \Lambda / 3$ first flips the norm of both lower helicities $( \pm 3 / 2, \pm 1 / 2)$ so the partially massless AdS theory cannot be unitary. Ironically, were negative values of $m^{2}$ not prohibited, we could traverse the lines in the correct order in dS. This observation lends weight to the speculation that the unitarity difficulties of partially massless theories are peculiar to half integer spins. Indeed, in the next Section we exhibit the bosonic $s=3$ example, which enjoys two partially massless unitary dS lines.

\subsection{Spin 3}

Spin 3 is the first example of a system with two new Bianchi identities over and above the usual one at $m^{2}=0$. The action and field equations (with 
flat space limits derived by KK descent in Appendix A.2) are

$$
\begin{gathered}
\mathcal{L}=\frac{1}{2} \sqrt{-g} \phi^{\mu \nu \rho} \mathcal{G}_{\mu \nu \rho}-\frac{3}{8} \sqrt{-g} \chi \mathcal{G}_{5} \\
\mathcal{G}_{\mu \nu \rho}=\left(\Delta^{(3)}-m^{2}+16 \Lambda / 3\right) \phi_{\mu \nu \rho}-3 D_{(\mu} D \cdot \phi_{\nu \rho)}+3 D_{(\mu} D_{\nu} \phi_{\rho) \sigma}{ }^{\sigma} \\
-3 g_{(\mu \nu}\left(\left(\Delta^{(1)}-m^{2}+11 \Lambda / 3\right) \phi_{\rho) \sigma}{ }^{\sigma}-D \cdot D \cdot \phi_{\rho) \sigma}{ }^{\sigma}+\frac{1}{2} D_{\rho)} D \cdot \phi_{\sigma}{ }^{\sigma}\right) \\
+\frac{3 m}{4} g_{(\mu \nu} D_{\rho)} \chi=0 \\
\mathcal{G}_{5}=\frac{3}{2}\left(\Delta^{(0)}-4 m^{2}+8 \Lambda\right) \chi+m D \cdot \phi_{\sigma}{ }^{\sigma}=0 .
\end{gathered}
$$

The field $\phi_{\mu \nu \rho}$ is a symmetric 3-tensor and the auxiliary field $\chi$ decouples at $m=0$ (the strictly massless theory). Fixing the ordering of covariant derivatives as shown and requiring constraints to remove all but the physical $7=2 s+1$ degrees of freedom, uniquely specifies all terms with an explicit $\Lambda$-dependence. Indeed, we find the following constraints

$$
\begin{aligned}
\mathcal{B}_{\{\nu \rho\}} & \equiv D \cdot \mathcal{G}_{\{\nu \rho\}}=-\frac{1}{2} m\left(D_{\{\nu} D_{\rho\}} \chi+2 m D \cdot \phi_{\{\nu \rho\}}-4 m D_{\{\nu} \phi_{\rho\} \sigma}{ }^{\sigma}\right), \\
\mathcal{B}_{\rho} & \equiv D \cdot \mathcal{B}_{\rho}-\frac{m}{4} D_{\rho} \mathcal{G}_{5}+\frac{m^{4}}{4} \mathcal{G}_{\rho \sigma}{ }^{\sigma}=\frac{5}{8} m\left(3 m^{2}-4 \Lambda\right)\left(D_{\rho} \chi+\frac{2}{3} m \phi_{\rho \sigma}{ }^{\sigma}\right), \\
\mathcal{B} & \equiv D \cdot \mathcal{B}-\frac{5}{12} m\left(3 m^{2}-4 \Lambda\right) \mathcal{G}_{5}=\frac{5}{2} m\left(3 m^{2}-4 \Lambda\right)\left(m^{2}-2 \Lambda\right) \chi .
\end{aligned}
$$

The explicit tensorial structures on the right hand sides of (90) are the covariantizations of the flat space ones in (129)-(131). The new phenomenon is the splitting of the prefactors to $m,\left(3 m^{2}-4 \Lambda\right)$ and $\left(m^{2}-2 \Lambda\right)$, thanks to the additional parameter $\Lambda$. Therefore, in addition to the usual massless theory at $m=0$ there are new gauge invariant systems at $m^{2}=4 \Lambda / 3$ and $m^{2}=2 \Lambda$, since whenever these prefactors vanish, the corresponding constraints in (90)-(92) become Bianchi identities with accompanying, respective, gauge invariances

$$
\delta \phi_{\mu \nu \rho}=D_{(\mu} \xi_{\{\nu \rho\})}, \quad \delta \chi=0
$$




$$
\begin{aligned}
& \delta \phi_{\mu \nu \rho}=D_{(\mu} D_{\{\nu} \xi_{\rho\})}+\frac{\Lambda}{3} g_{(\mu \nu} \xi_{\rho)}, \quad \delta \chi=-\frac{2}{3} \sqrt{\frac{\Lambda}{3}} D . \xi \\
& \delta \phi_{\mu \nu \rho}=D_{(\mu} D_{\{\nu} D_{\rho\})} \xi+\frac{\Lambda}{2} g_{(\mu \nu} D_{\rho)} \xi, \quad \delta \chi=-\frac{2}{3} \sqrt{\frac{\Lambda}{2}}\left(D^{2}+\frac{10 \Lambda}{3}\right) \xi .
\end{aligned}
$$

The new gauge invariant lines bound regions in the $\left(m^{2}, \Lambda\right)$ half-plane whose unitarity properties are determined by the signs of the prefactors $\left(3 m^{2}-4 \Lambda\right)$ and $\left(m^{2}-2 \Lambda\right)$. To analyze these new properties, decompose the $7=2 s+1$ physical DoF into helicities $( \pm 3, \pm 2, \pm 1,0)$. We find the following "phase" structure of the $\left(m^{2}, \Lambda\right)$ half-plane

- $\underline{m}^{2}>2 \Lambda>0$ : This region includes Minkowski space and is clearly unitary. All helicities $( \pm 3, \pm 2, \pm 1,0)$, propagate with positive norm.

- $\underline{m}^{2}=2 \Lambda$ : A partially massless theory appears since the scalar constraint $\mathcal{B}=0$ is now a Bianchi identity whose associated gauge invariance removes the scalar helicity 0 excitation. The remaining 6 DoF, $( \pm 3, \pm 2, \pm 1)$, propagate with positive norm since they are unaffected by the scalar gauge invariance.

- $4 \Lambda / 3<m^{2}<2 \Lambda$ : Although all 7 DoF are now again propagating, the scalar helicity 0 mode reemerges from the gauge boundary $m^{2}=2 \Lambda$ with negative norm (since the factor $\left(3 m^{2}-4 \Lambda\right)\left(m^{2}-2 \Lambda\right)$ appears in canonical commutators as a negative denominator). This is a unitarily forbidden region.

- $m^{2}=4 \Lambda / 3$ : This partially massless theory has Bianchi identities $\mathcal{B}=$

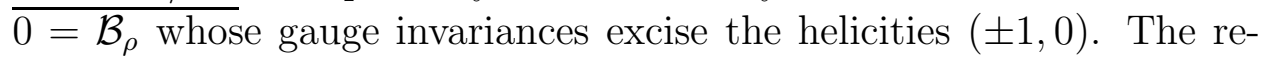
maining $4 \mathrm{PDoF}$, helicities $( \pm 3, \pm 2)$ propagate with positive norm.

- $0<m^{2}<4 \Lambda / 3$ : Again all 7 DoF propagate but now the scalar helicity has again positive norm since its denominator $\left(3 m^{2}-4 \Lambda\right)\left(m^{2}-2 \Lambda\right)$ is again positive. However, the region is still unitarily forbidden because now the vector helicities \pm 1 suffer a negative denominator $\left(3 m^{2}-4 \Lambda\right)$.

- $\underline{m}^{2}=0$ : This is the unitary strictly massless model with tensor Bianchi identity $\mathcal{B}_{\{\nu \rho\}}=0=\mathcal{B}_{\rho}=\mathcal{B}$. Only the uppermost helicities \pm 3 remain. An added subtlety is the remnant decoupled auxiliary field $\chi$. 
Notice how the uppermost helicity \pm 3 always emerges unscathed as a pair of positive norm states but, unlike Minkowski space, splittings into theories with intermediate lower helicities, rather than only the full complement of $2 s+1$ states of the massive theory, are possible. Furthermore, the ordering of gauge lines is the same as for the $s=5 / 2$ example. But, unlike for $s=5 / 2$, the lines can be traversed in the order required for unitarity of the partially massless dS theories without recourse to unphysical, negative, values of $m^{2}$.

\section{Conclusions}

Life in cosmological backgrounds [19 promises to be less degenerate than its Minkowski limit. In this paper, we have examined the notion of mass for higher spins in (A)dS geometries and found a far richer structure than in flat space, where higher spin fields are either strictly massless or massive. Once a cosmological constant is present, the Minkowski gauge invariances implying masslessness split into a set of partial ones allowing intermediate, partially massless theories along lines in the $\left(\mathrm{m}^{2}, \Lambda\right)$ plane. Their unitarity is easily analyzed by examining the line ordering. Although interactions of relativistic higher spin fields are fraught with many difficulties】 even in flat backgrounds, and the observed cosmological scales are far removed from those encountered in (for example) nuclear physics, this new phenomenon is rather fascinating. However, many open questions remain, some of which are:

Perhaps the most pressing is an explicit generalization of our results, beyond simple counting arguments, to generic values of $s$. It is clear that partial masslessness applies to higher values of $s$ when $m^{2}$ is appropriately tuned to $\Lambda$, thereby converting massive constraints to Bianchi identities. Yet a simple formalism for deducing the pertinent tunings of of $\mathrm{m}^{2}$ would be extremely useful since the unitarity properties of these partially massless theories can then be deduced by inspection by studying the ordering of the gauge lines. Although this problem may appear technically challenging, an interesting approach is suggested by the flat space KK descent methods presented in the Appendix.

Detailed knowledge of models with $s>3$ would reveal whether the failure of the $s=5 / 2$ partially massless theories to be unitary is a universal fermionic characteristic. This question is also related to the lack of unitary supergravity

\footnotetext{
${ }^{7}$ For massless fields, it has been suggested that these problems can be alleviated in AdS backgrounds 20].
} 
theories in $\mathrm{dS}$ spaces [18]. One would also like to know whether auxiliary fields of the massless theories decouple, not only in the strictly massless limit, but also partially massless ones.

Another interesting issue is that of the higher spin version of the $s=2$ van Dam-Veltman-Zhakarov discontinuity for massless limits of massive $s=2$ exchange processes [21]. Clearly, if higher spins are allowed to run in loops, there will generically be discontinuities thanks to the jump in the virtual DoF over which one functionally integrates [22]. A much simpler physical problem, whose eikonal limit is purely classical, is that of exchange processes mediated by massive higher spins. For $s=2$, the flat space discontinuity of the massless limit can be cured by taking the limit in a $\Lambda \neq 0$ background [23]. The same flat space difficulty extends to $s=3 / 2$ [24] but is again removed in a cosmological background [11]. The same question can be posed for higher $s$ both in strictly and partially massless limits.

An interesting feature of the $m^{2}=2 \Lambda / 3$ partially massless $s=2$ theory is that the remaining helicity $( \pm 2, \pm 1)$ excitations propagate on the null cone of the conformally flat dS metric [1]. Do partially massless $s>2$ theories also exhibit this conformal behavior?

\section{A Appendix: Massive Constraints from Di- mensional Reduction of Bianchi Identities}

The (A)dS higher spin wave equations presented in this paper were obtained by starting with their flat space antecedents, coupling minimally to gravity and then adding the (unique) non-minimal terms required for constraints to produce the correct PDoF count. Although both massless [26, 6] and massive [27] higher spin Minkowski field equations are available, for our purposes a derivation simultaneously yielding the massive constraints is desirable. Fortunately, dimensional reduction provides the desired derivation of the massive field equations from their simpler, $d=5$ massless ancestors $[8]$. The key idea is that a massless field has the same DoF count as a massive one in one dimension lower. In this Appendix, we summarize the dimensional reduction method and show, in flat space, that it allows the massive constraints to be derived from the Bianchi identities of the massless theories. [It would be

\footnotetext{
${ }^{8}$ Curiously the limit $m \rightarrow 0$, to a non-unitary massive theory, in AdS is also continuous [25].
} 
most interesting to rederive the $(\mathrm{A}) \mathrm{dS}$ results of our paper by dimensional reduction in a curved background, a problem we reserve for future investigations.] The methods reported here for $s=5 / 2$ and $s=3$, of course, apply equally well to other values of $s$.

\section{A.1 Free Massive Spin 5/2}

The massless $s=5 / 2$ action and field equations are

$$
\begin{gathered}
\mathcal{L}=-\frac{1}{2} \bar{\psi}^{M N} \mathcal{R}_{M N} \\
\mathcal{R}_{M N}=\widehat{\partial} \psi_{M N}-2 \Gamma_{(M} \partial . \psi_{N)}-2 \partial_{(M} \Gamma \cdot \psi_{N)}+2 \Gamma_{(M} \widehat{\partial} \Gamma \cdot \psi_{N)} \\
+\eta_{M N} \Gamma \cdot \partial \cdot \psi+\left(\partial_{(M} \Gamma_{N)}-\frac{1}{2} \eta_{M N} \widehat{\partial}\right) \psi_{R}{ }^{R}=0
\end{gathered}
$$

where, in $d=5, M=(\mu, 5)$ and Dirac matrices $\Gamma_{M}$ are defined analogously to our $d=4$ conventions and $\widehat{X} \equiv \Gamma . X$. The action enjoys the gauge invariance

$$
\delta \Psi_{M N}=\partial_{(M} \varepsilon_{N)}
$$

subject to

$$
\Gamma . \varepsilon=0
$$

thanks to the Bianchi identity

$$
\partial . \mathcal{R}_{N}=\frac{1}{5} \Gamma_{N} \Gamma . \partial . \mathcal{R}
$$

Before proceeding, observe that the massless field equations (98) in $d=5$ can be rewritten in the simple Christoffel form [6, 28]

$$
\mathcal{C}_{M N} \equiv \mathcal{R}_{M N}-\frac{1}{5} \eta_{M N} \mathcal{R}_{R}{ }^{R}-\frac{2}{5} \Gamma_{(M} \Gamma \cdot \mathcal{R}_{N)}=\widehat{\partial} \psi_{M N}-2 \partial_{(M} \Gamma \cdot \psi_{N)}=0 .
$$

We now dimensionally reduce: First, we would like to gauge away components carrying the index "5", so examine the gauge transformations

$$
\begin{aligned}
\delta \psi_{55} & =\partial_{5} \varepsilon_{5}, \\
\delta \psi_{5 \mu} & =\frac{1}{2}\left(\partial_{5} \varepsilon_{\mu}+\partial_{\mu} \varepsilon_{5}\right) .
\end{aligned}
$$


Assuming that $\partial_{5}$ is invertible, we replace

$$
\partial_{5} \rightarrow i m
$$

where $m$ will be the $d=4$ mass. Hence, $\psi_{55}$ may be gauged away. However the gamma-trace condition on the gauge parameter $\Gamma . \epsilon=0$ prevents us from doing the same to all components of $\psi_{5 \mu}$. [This is the reason [8] auxiliary fields cannot be avoided beyond $s=2$.] Instead, we choose a gauge in which only the $(d=4)$ gamma-traceless components are removed

$$
\psi_{5 \mu}-\frac{1}{4} \gamma_{\mu} \gamma \cdot \psi_{5} \equiv \psi_{5 \tilde{\mu}}=0, \quad \psi_{55}=0
$$

where the $d=4$ Dirac matrices are

$$
\gamma_{\mu} \equiv i \Gamma_{\mu} \Gamma_{5}, \quad \gamma_{5} \equiv \Gamma_{5}
$$

In terms of the auxiliary Dirac spinor

$$
\chi \equiv-i \gamma^{\mu} \psi_{5 \mu} \Longrightarrow \psi_{5 \mu}=\frac{i}{4} \gamma_{\mu} \chi
$$

the equations of motion (102) read

$$
\begin{aligned}
-i \Gamma_{5} \mathcal{C}_{\mu \nu} & =(\not \partial+m) \psi_{\mu \nu}-2 \partial_{(\mu}\left(\gamma \cdot \psi_{\nu)}+\frac{1}{4} \gamma_{\nu)} \chi\right)=0 \\
-i \Gamma_{5} \mathcal{C}_{5 \mu} & =-i\left(m \gamma \cdot \psi_{\mu}+\frac{1}{2}\left(\partial_{\mu}+\frac{1}{2} \gamma_{\mu} \not \partial\right) \chi\right)=0 \\
-i \Gamma_{5} \mathcal{C}_{55} & =2 m \chi=0
\end{aligned}
$$

they imply

$$
(\not \partial+m) \psi_{\mu \nu}=0, \quad \gamma \cdot \psi_{\mu}=0 \Rightarrow \partial \cdot \psi_{\mu}=0=\psi,
$$

\footnotetext{
${ }^{9}$ Conversely, it may be tempting to gauge away $\psi_{5 \mu}$ and retain $\psi_{55}$ as an auxiliary field. This gauge choice does not completely fix the gauge, and the resulting system of equations is invariant under residual gauge transformations $\delta \psi_{55}=2 m \alpha$ and $\delta \psi_{\mu \nu}=$ $(1 / 2 m) \partial_{\mu} \partial_{\nu} \alpha$ where the gauge parameter satisfies $(\not \partial+m) \alpha=0$. One obtains the correct DoF upon fixing this remaining freedom. In fact there are a wide range of (residual) gauge invariant equations describing a massive $s=5 / 2$ field depending on the details of the gauge choice made. The choice (106) has the advantage of completely fixing the gauge freedom algebraically.
} 
These are the usual on-shell conditions for a massive $d=4, s=5 / 2$ field. In particular observe that we can identify the constraints as $\mathcal{C}_{5 \widetilde{\mu}}$ and $\mathcal{C}_{55}$. We stress that the field equations in their simplest form (109)-(111) do not directly follow from the variation of an action. However the massive $d=4$ action can be obtained from its $d=5$ massless counterpart by imposing the gauge condition (106) and performing the KK descent:

$$
\mathcal{L}=-\frac{1}{2} \bar{\psi}^{\mu \nu} \mathcal{R}_{\mu \nu}-\frac{i}{4} \bar{\chi} \gamma^{\mu} \mathcal{R}_{5 \mu}
$$

Here we have relabeled $-i \Gamma_{5} \mathcal{R}_{M N} \rightarrow \mathcal{R}_{M N}$ and $\bar{\psi}^{\mu \nu} \equiv \psi^{\dagger \mu \nu} i \gamma^{0}$ is the $d=4$ Dirac conjugate (remember that $\Gamma^{0}=-i \gamma^{0} \Gamma^{5}$ ). The field equations are

$$
\begin{aligned}
\mathcal{R}_{\mu \nu} & =\not \partial \psi_{\mu \nu}-2 \gamma_{(\mu} \partial . \psi_{\nu}-2 \partial_{(\mu} \gamma \cdot \psi_{\nu)}+2 \gamma_{(\mu} \not \partial \cdot \psi_{\nu)} \\
& +\eta_{\mu \nu} \gamma \cdot \partial \cdot \psi+\left(\partial_{(\mu} \gamma_{\nu)}-\frac{1}{2} \not \partial\right)\left(\psi_{\rho}{ }^{\rho}+\frac{1}{2} \chi\right) \\
& +m\left(\psi_{\mu \nu}-2 \gamma_{(\mu} \gamma \cdot \psi_{\nu)}-\frac{1}{2} \eta_{\mu \nu} \psi_{\rho}{ }^{\rho}\right)+m \eta_{\mu \nu} \chi=0, \\
\frac{i}{4} \gamma^{\mu} \mathcal{R}_{5 \mu} & =-\frac{1}{2}(\not \partial-2 m) \chi+\frac{1}{4} \partial \cdot \gamma \cdot \psi-\frac{1}{8}(\not \partial+4 m) \psi=0 .
\end{aligned}
$$

After the field redefinition

$$
\psi_{\mu \nu} \rightarrow \psi_{\mu \nu}-\frac{1}{8} \eta_{\mu \nu} \chi
$$

the auxiliary field $\chi$ couples only via $m \bar{\chi} \psi_{\rho}{ }^{\rho}$ and the field equations are then the flat limit of the ones presented in (77) and (78).

Finally, we show how to deduce the constraints $\mathcal{C}_{5 \tilde{\mu}}$ and $\mathcal{C}_{55}$ from the Bianchi identity (101): We need the remaining components $\mathcal{R}_{5 \tilde{\mu}}$ and $\mathcal{R}_{55}$ of $\mathcal{R}_{M N}$, since the complete set $\mathcal{R}_{M N}$ inserted in (102) provides the equations $\mathcal{C}_{M N}$. The Bianchi identity (101) rewritten as

$$
i m \mathcal{R}_{5 N}=-\partial^{\mu} \mathcal{R}_{\mu N}+\frac{1}{5} \Gamma_{N} \partial . \Gamma . \mathcal{R}
$$

can be used to "reconstitute" the missing equations, in particular

$$
\mathcal{R}_{5 \widetilde{\nu}}=\frac{i}{m} \partial^{\mu} \mathcal{R}_{\mu \widetilde{\nu}}
$$

and in turn

$$
\mathcal{R}_{55}=-\frac{1}{m^{2}} \partial^{\rho} \partial^{\sigma} \mathcal{R}_{\rho \sigma}+\frac{1}{4 m^{2}}(\not \partial-m)\left(\partial^{\rho} \gamma^{\sigma} \mathcal{R}_{\rho \sigma}+i m \gamma^{\rho} \mathcal{R}_{5 \rho}\right)
$$


Clearly, since (117) is an identity, the system of equations (114) and (115) along with (118) and (119) are guaranteed to be equivalent to (109)-(111). when inserted in the definition (102). It is easy to verify that this calculation combined with the field redefinition (116) yields the flat limit of the constraints (80) and (82). Incidentally, this proves that the action (113) describes the correct $2 s+1=6 \mathrm{PDoF}$.

\section{A.2 Free Massive Spin 3}

The $s=3, d=5$ massless Lagrangian and field equations are

$$
\begin{gathered}
\mathcal{L}=\frac{1}{2} \phi_{M N R} \mathcal{G}^{M N R}, \\
\mathcal{G}_{M N R}=\square_{5} \phi_{M N R}-3 \partial_{(M} \partial . \phi_{N R)}+3 \partial_{(M} \partial_{N} \phi_{R) S}{ }^{S} \\
-3 \eta_{(M N}\left(\square_{5} \phi_{R) S}{ }^{S}-\partial . \partial . \phi_{R) S}{ }^{S}+\frac{1}{2} \partial_{R)} \partial . \phi_{S}{ }^{S}\right)=0 .
\end{gathered}
$$

The action (120) has the gauge invariance

$$
\delta \phi_{M N R}=\partial_{(M} \xi_{N R)},
$$

with the restriction

$$
\xi_{N}{ }^{N}=0,
$$

corresponding to the Bianchi identity

$$
\partial \cdot \mathcal{G}_{M N}=\frac{1}{5} \eta_{M N} \partial \cdot \mathcal{G}_{R}{ }^{R} .
$$

Again, there is a simpler Christoffel form for the field equations [6]

$$
\begin{aligned}
\mathcal{B}_{M N R} & \equiv \mathcal{G}_{M N R}-\frac{3}{5} \eta_{(M N} \mathcal{G}_{R) S}{ }^{S} \\
& =\square_{5} \phi_{M N R}-3 \partial_{(M} \partial . \phi_{N R)}+3 \partial_{(M} \partial_{N} \phi_{R) S}{ }^{S}=0 .
\end{aligned}
$$

The massive field content is obtained by algebraically gauging away the components

$$
\phi_{555}=0, \quad \phi_{\mu 55}=0, \quad \phi_{5\{\mu \nu\}} \equiv \phi_{5 \mu \nu}-\frac{1}{4} \eta_{\mu \nu} \phi_{5 \rho}{ }^{\rho}=0 .
$$


There are no residual gauge invariances; the remaining fields are $\phi_{\mu \nu \rho}$ and the scalar auxiliary

$$
\chi \equiv-i \phi_{5 \mu}{ }^{\mu} \Rightarrow \phi_{\mu \nu 5}=\frac{i}{4} \eta_{\mu \nu} \chi
$$

Upon KK descent, $\partial_{5} \rightarrow i m$ and the field equations (125) become

$$
\begin{aligned}
\mathcal{B}_{\mu \nu \rho} & =\left(\square-m^{2}\right) \phi_{\mu \nu \rho}-3 \partial_{(\mu} \partial . \phi_{\nu \rho)}+3 \partial_{(\mu} \partial_{\nu} \phi_{\rho) \sigma}{ }^{\sigma}+\frac{3 m}{4} \eta_{(\mu \nu} \partial_{\rho)} \chi \\
\mathcal{B}_{\mu \nu 5} & =\frac{i}{4} \eta_{\mu \nu} \square \chi+\frac{i}{2} \partial_{\mu} \partial_{\nu} \chi-i m\left(\partial . \phi_{\mu \nu}-2 \partial_{(\mu} \phi_{\nu) \sigma}{ }^{\sigma}\right) \\
\mathcal{B}_{\mu 55} & =-\frac{3}{2} m \partial_{\mu} \chi-m^{2} \phi_{\mu \sigma}{ }^{\sigma} \\
\mathcal{B}_{555} & =-3 i m^{2} \chi
\end{aligned}
$$

from which the usual massive $s=3$ on-shell conditions follow immediately,

$$
\left(\square-m^{2}\right) \phi_{\mu \nu \rho}=0, \quad \chi=0=\phi_{\mu}=\partial . \phi_{\mu \nu} .
$$

Clearly $\mathcal{B}_{\{\mu \nu\} 5}, \mathcal{B}_{\mu 55}$ and $\mathcal{B}_{555}$ are constraints. The $d=4$ massive action principle, guaranteed to yield (128)-(131) by virtue of the Bianchi identity, is

$$
\mathcal{L}=\frac{1}{2} \phi^{\mu \nu \rho} \mathcal{G}_{\mu \nu \rho}+\frac{3 i}{8} \chi \mathcal{G}_{5 \mu}{ }^{\mu}
$$

The $d=4$ massive field equations read

$$
\begin{aligned}
\mathcal{G}_{\mu \nu \rho}= & \left(\square-m^{2}\right) \phi_{\mu \nu \rho}-3 \partial_{(\mu} \partial . \phi_{\nu \rho)}+3 \partial_{(\mu} \partial_{\nu} \phi_{\rho)}+\frac{3 m}{4} \eta_{(\mu \nu} \partial_{\rho)} \chi \\
& -3 \eta_{(\mu \nu}\left(\left(\square-m^{2}\right) \phi_{\rho)}-\partial . \partial . \phi_{\rho)}+\frac{1}{2} \partial_{\rho} \partial . \phi\right) \\
\frac{3 i}{4} \mathcal{G}_{5}= & \frac{9}{8}\left(\square-4 m^{2}\right) \chi+\frac{3 m}{4} \partial . \phi .
\end{aligned}
$$

These agree with the flat limit of (88) and (89). The Bianchi identity (124) can be rewritten as

$$
i m \mathcal{G}_{5 M N}=-\partial^{\rho} \mathcal{G}_{\rho M N}+\frac{1}{5} \eta_{M N} \partial \cdot \mathcal{G}_{R}{ }^{R}
$$


and yields the "missing" field equations

$$
\begin{aligned}
\mathcal{G}_{\{\mu \nu\} 5} & =\frac{i}{m} \partial^{\rho} \mathcal{G}_{\rho\{\mu \nu\}} \\
\mathcal{G}_{\mu 55} & =-\frac{1}{m^{2}}\left(\partial^{\rho} \partial^{\sigma} \mathcal{G}_{\sigma\{\rho \mu\}}-\frac{i m}{4} \partial_{\mu} \mathcal{G}_{5}\right) \\
\mathcal{G}_{555} & =-\frac{i}{m^{3}} \partial^{\mu} \partial^{\nu} \partial^{\sigma} \mathcal{G}_{\sigma\{\mu \nu\}}-\frac{1}{4 m^{2}}\left(\square-m^{2}\right) \mathcal{G}_{5}-\frac{i}{4 m} \partial^{\rho} \mathcal{G}_{\rho \sigma}{ }^{\sigma} .
\end{aligned}
$$

Substituting these in the constraints $\mathcal{B}_{\{\mu \nu\} 5}, \mathcal{B}_{\mu 55}$ and $\mathcal{B}_{555}$ following from (125) yields the flat limit of the ones given in (90)-(92).

\section{Acknowledgments}

This work was supported by the National Science Foundation under grant PHY99-73935.

\section{References}

[1] S. Deser and R. I. Nepomechie, Phys. Lett. B 132, 321 (1983); Annals Phys. 154, 396 (1984).

[2] A. Higuchi, Nucl. Phys. B 282, 397 (1987); ibid 325, 745 (1989); J. Math. Phys. 28, 1553 (1987).

[3] S. Deser and A. Waldron, "Gauge invariances and phases of massive higher spins in (A)dS," hep-th/0102166.

[4] K. Johnson and E. C. Sudarshan, Annals Phys. 13, 126 (1961).

[5] G. Velo and D. Zwanziger, Phys. Rev. 186, 1337 (1969); A. Shamaly and A. Z. Capri, Ann. Phys. 74 503, (1972). S. Deser, V. Pascalutsa and A. Waldron, Phys. Rev. D62, 105031 (2000) hep-th/0003011.

[6] B. de Wit and D. Z. Freedman, Phys. Rev. D 21, 358 (1980).

[7] A. Lichnerowicz, Institut des Hautes Études Scientifiques, 10, 293 (1961); Bull. Soc. Math. France, 92, 11 (1964).

[8] C. Aragone, S. Deser and Z. Yang, Annals Phys. 179, 76 (1987). 
[9] D. G. Boulware and S. Deser, Phys. Rev. D6, 3368 (1972).

[10] I. L. Buchbinder, D. M. Gitman, V. A. Krykhtin and V. D. Pershin, Nucl. Phys. B584, 615 (2000) hep-th/9910188]; I. L. Buchbinder, D. M. Gitman and V. D. Pershin, Phys. Lett. B 492, 161 (2000) hepth/0006144.

[11] S. Deser and A. Waldron, Phys. Lett. B 501, 134 (2001) hepth/0012014.

[12] P. K. Townsend, Phys. Rev. D 15, 2802 (1977); S. Deser and B. Zumino, Phys. Rev. Lett. 38, 1433 (1977).

[13] E. Witten, talk presented Strings 2001, Tata Institute, Mumbai 2001.

[14] B. S. DeWitt and R. W. Brehme, Annals Phys. 9, 220 (1960); Phys. Rev. 4, 317 (1960).

[15] S. Deser and A. Waldron, "Stability of Massive Cosmological Gravitons", hep-th/0103255.

[16] I. Bengtsson, J. Math. Phys. 36, 5805 (1995) gr-qc/9411057.

[17] L. F. Abbott and S. Deser, Nucl. Phys. B 195, 76 (1982).

[18] K. Pilch, P. van Nieuwenhuizen and M. F. Sohnius, Commun. Math. Phys. 98, 105 (1985).

[19] P. Riess, et al., Astron. J. 116, 1009 (1998) astro-ph/9805021; P. M. Garnavich, et al., Astrophys. J. 509, 74 (1998) astro-ph/9806391; S. Perlmutter, et al., Nature 391, 51 (1998) astro-ph/9712212.

[20] M. A. Vasiliev, "Higher spin symmetries, star-product and relativistic equations in AdS space," hep-th/0002183; L. Brink, R. R. Metsaev and M. A. Vasiliev, Nucl. Phys. B 586, 183 (2000) hep-th/0005136.

[21] H. van Dam and M. Veltman, Nucl. Phys. B22, 397 (1970); V. I. Zakharov, JETP Lett. 12, 312 (1970); L. D. Faddeev and A. A. Slavnov, Theor. Math. Phys. 3, 18 (1970); S. K. Wong, Phys. Rev. D3, 945 (1971). 
[22] F. A. Dilkes, M. J. Duff, J. T. Liu and H. Sati, "Quantum $M \longrightarrow 0$ ] discontinuity for massive gravity with a Lambda term," hep-th/0102093.

[23] I. I. Kogan, S. Mouslopoulos and A. Papazoglou, "The $m \longrightarrow 0$ limit for massive graviton in $\mathrm{dS}(4)$ and $\mathrm{AdS}(4)$ : How to circumvent the van DamVeltman-Zakharov discontinuity," hep-th/0011138; M. Porrati, Phys. Lett. B 498, 92 (2001) hep-th/0011152].

[24] S. Deser, J. H. Kay and K. S. Stelle, Phys. Rev. D16, 2448 (1977).

[25] P. A. Grassi and P. van Nieuwenhuizen, Phys. Lett. B 499, 174 (2001) hep-th/0011278.

[26] C. Fronsdal, Phys. Rev. D 18, 3624 (1978); J. Fang and C. Fronsdal, Phys. Rev. D 18, 3630 (1978); T. Curtright, Phys. Lett. B 85, 219 (1979);

[27] L. P. Singh and C. R. Hagen, Phys. Rev. D 9, 910 (1974); ibid 898 (1974). S. -J. Chang, Phys. Rev. 161, 1308 (1967). F. A. Berends, J. W. van Holten, P. van Nieuwenhuizen and B. de Wit, Nucl. Phys. B 154, 261 (1979).

[28] T. Damour and S. Deser, Annales Poincare Phys. Theor. 47, 277 (1987). 